\title{
Sobolev maps on manifolds: degree, approximation, lifting
}

\author{
Petru Mironescu*
}

January 20th, 2007

\begin{abstract}
In this paper, we review some basic topological properties of the space $X=W^{s, p}(M ; N)$, where $M$ and $N$ are compact Riemannian manifold without boundary. More specifically, we discuss the following questions: can one define a degree for maps in $X$ ? are smooth or not-farfrom-being-smooth maps dense in $X$ ? can one lift $\mathbb{S}^{1}$-valued maps?
\end{abstract}

\section{Introduction}

Sobolev maps between manifolds appear naturally in different contexts: harmonic maps, liquid crystals and the Ginzburg-Landau equation are some of them. In this survey, we describe what is known about three natural questions concerning maps $u: M \rightarrow N$ in a Sobolev space $X=W^{s, p}(M ; N)$; here, $M$ and $N$ are compact Riemannian manifold without boundary. Question 1 . Does $u$ has a degree? Question2. Can one approximate $u$ with smooth $N$-valued maps? Question 3. If $N=\mathbb{S}^{1}$, can one write $u=e^{\imath \varphi}$, with $\varphi$ as smooth as $u$ ? Though these questions are motivated by applications, this paper is rather a quick introduction to research problems than applications oriented.

Degree is discussed in Section 2. Sections 2.6 and 2.7 may be viewed as an introduction to the problem of describing the connected components of $X$; this is beyond the scope of this paper, and we send the reader to the beautiful papers [BL] and [HaL2]. Section 3 deals with lifting. Section 4 is about approximation with smooth maps. This matter is the source of additional research directions; some of them will be addressed in Sections 4.3-4.4. We sometimes go beyond Sobolev spaces and investigate properties of VMO or BV maps.

The aim of this paper is to give a quick overview of the existent literature. Therefore, proofs are rather sketchy; often, "hint" would have been more appropriate than "proof". However, we hope that the main ideas are sufficiently clearly exposed. On the other hand, the bibliography is reduced to the strict minimum; it contains only the papers that were quoted in the text.

Part of the open problems is not new. Some of them are directly inspired by the surveys [B1], [B2]. Others raised from useful discussions with Haïm Brezis, to whom the author wants to express his gratitude.

\section{$1.1 \quad$ Notations}

For $0<s<\infty$ and $1 \leq p<\infty$, we equip the Sobolev space $W^{s, p}$ with the norm $u \mapsto\|u\|_{L^{p}}+|u|_{W^{s, p}}$. If $s$ is an integer, $|u|_{W^{s, p}}=\left\|D^{s} u\right\|_{L^{p}}$. If not, write $s=m+\sigma$, with $m$ integer and $0<\sigma<1$. Then,

*Institut Camille Jordan, Université Lyon 1 UMR 5208, 43 boulevard du 11 novembre 1918, 69622 Villeurbanne cedex France. Email address: mironescu@math.univ-lyon1.fr 
in $\mathbb{R}^{n}$ or on an $n$-dimensional manifold,

$$
|u|_{W^{s, p}}^{p}=\iint \frac{\left|D^{m} u(x)-D^{m} u(y)\right|^{p}}{|x-y|^{n+\sigma p}} d x d y .
$$

In $\mathbb{R}^{n}$, the BMO norm is $\|u\|_{\mathrm{BMO}}=\|u\|_{L^{1}}+|u|_{\mathrm{BMO}}$, where

$$
|u|_{\mathrm{BMO}}=\sup _{x} \sup _{r>0} f_{B(x, r)} f_{B(x, r)}|u(y)-u(z)| d y d z .
$$

When $\mathbb{R}^{n}$ is replaced by an embedded manifold $M$, averages are computed on $B(x, r) \cap M$.

In $\mathrm{BV}$, we consider the norm $\|u\|_{\mathrm{BV}}=\|u\|_{L^{1}}+|u|_{\mathrm{BV}}$, where $|u|_{\mathrm{BV}}$ is the total variation of the measure Du.

If $N$ is a compact manifold embedded into $\mathbb{R}^{l}$ and $M$ is any smooth manifold, then $W^{s, p}(M ; N)=$ $\left\{u: M \rightarrow \mathbb{R}^{l} ; u(x) \in N\right.$ a. e. and $\left.|u|_{W^{s, p}}<\infty\right\}$. One defines similarly $\operatorname{BV}(M ; N)$ and $\operatorname{BMO}(M ; N)$. $\mathbb{S}^{n}$ is the unit sphere in $\mathbb{R}^{n+1}$. $B^{n}$ is the unit ball in $\mathbb{R}^{n}$.

On a compact $n$-dimensional manifold $M$ embedded in an Euclidean space, a mollifier is a $C^{\infty}$-map $(0, \infty) \times M \times M \ni(\varepsilon, x, y) \mapsto \rho_{\varepsilon}(x, y)$ such that supp $\rho_{\varepsilon}(x, \cdot) \subset B(x, \varepsilon) \cap M, \int \rho_{\varepsilon}(x, y) d y=1$ and $\left\|D^{j} \rho_{\varepsilon}\right\| \leq C \varepsilon^{-n-j}$. With some abuse of notations, we will denote $\int u(y) \rho_{\varepsilon}(x, y) d y$ by $u * \rho_{\varepsilon}(x)$.

\section{Maps from $\mathbb{S}^{n}$ into $\mathbb{S}^{n}$}

\subsection{Degree}

In this part, $X=W^{s, p}\left(\mathbb{S}^{n} ; \mathbb{S}^{n}\right)$. The basic question we address is: can one define a degree for maps in $X$ ? If we have in mind the way the topological degree is defined for continuous maps, the natural strategy would be: Step 1. Prove that $\mathbb{S}^{n}$-valued smooth maps are dense in $X$; Step 2. Prove that the degree, initially defined for smooth maps, extends by density to the whole $X$.

It turns out that Step 1 works.

Theorem 2.1. $C^{\infty}\left(\mathbb{S}^{n} ; \mathbb{S}^{n}\right)$ is dense in $X$ for each $s$ and $p$.

Proof. When $s p>n$ or $s=n$ and $p=1, X$ is embedded in $C^{0}$, by the Sobolev embeddings. In this case, the proof is trivial: approximate $u \in X$ by a smooth family $u_{\varepsilon}=u * \rho_{\varepsilon}$. Then $\left|u_{\varepsilon}\right| \rightarrow 1$ uniformly. For sufficiently small $\varepsilon$, take $v_{\varepsilon}=u_{\varepsilon} /\left|u_{\varepsilon}\right|$. It is easy to see that $v_{\varepsilon} \rightarrow u$ in $X$.

When $s p<n$, this result is due to Escobedo [E] when $s$ is not an integer; however, his proof adapts to the case where $s$ is an integer. Here it is how it works: fix any point $x$ in $\mathbb{S}^{n}$ and consider, for small $\varepsilon>0$, a smooth map $\pi=\pi_{x, \varepsilon}: \mathbb{S}^{n} \rightarrow \mathbb{S}^{n} \backslash B(x, \varepsilon)$ projecting the cap $B(x, \varepsilon) \cap \mathbb{S}^{n}$ onto its complement in $\mathbb{S}^{n}$. This can be done such that $\pi=$ id outside $B(x, 2 \varepsilon) \cap \mathbb{S}^{n}$ and $\left\|D^{k} \pi\right\| \leq C \varepsilon^{-k}$. If $u \in X$, then $v=v_{x, \varepsilon}=\pi \circ u$ is still in $X$ and has the additional property that it can be approximated by $\mathbb{S}^{n}$-valued smooth maps. Indeed, $v$ takes values into $\mathbb{S}^{n} \backslash B(x, \varepsilon)$, which is topologically like a ball. By composing $v$ with an appropriate diffeomorphism, we are in the situation where the map is $\mathbb{R}^{n}$-valued, and then approximation is standard. It remains to prove that, if we pick appropriately $\varepsilon \rightarrow 0$ and $x=x(\varepsilon)$, then the corresponding $v$ 's converge to $u$. Here it is where the hypothesis $s p<n$ comes into the picture: it implies that $\int_{\mathbb{S}^{n}}\left\|v_{x, \varepsilon}-u\right\|_{W^{s, p}}^{p} d x \rightarrow 0$ as $\varepsilon \rightarrow 0$. This leads immediately to the desired conclusion.

When $s p=n$, the idea of the proof goes back to Schoen and Uhlenbeck [SU]; their proof works for 
$W^{1, n}\left(\mathbb{S}^{n} ; \mathbb{S}^{n}\right)$ maps. The general case is due to Boutet de Monvel and Gabber (Appendix to [BGP]). The starting point is that a map $u$ in $X$ belongs to VMO (=vanishing mean oscillation=the closure of smooth maps in BMO). Analytically, this means that, with $I_{\delta}(u)=\sup _{x \in \mathbb{S}^{n}} f_{B(x, \delta) \cap \mathbb{S}^{n}} f_{B(x, \delta) \cap \mathbb{S}^{n}} \mid u(y)-$ $u(z) \mid d y d z$, we have

$$
\lim _{\delta \rightarrow 0} I_{\delta}(u)=0 .
$$

(For a proof of the Sobolev embedding $W^{s, p}\left(\mathbb{R}^{n}\right) \hookrightarrow$ VMO when $s p=n$, see, e. g., [BNI], Section I.2). Next, the key ingredient is that, for $\operatorname{VMO}\left(\mathbb{S}^{n} ; \mathbb{S}^{n}\right)$ maps, we have

$$
\left|u_{\varepsilon}\right| \rightarrow 1 \text { uniformly as } \varepsilon \rightarrow 0 .
$$

Indeed, if $y \in B(x, \varepsilon) \cap \mathbb{S}^{n}$, then $\left|u(y)-u_{\varepsilon}(x)\right| \leq C f_{B(x, \varepsilon) \cap \mathbb{S}^{n}}|u(y)-u(z)| d z$. Thus

$$
1-\left|u_{\varepsilon}(x)\right|=f_{B(x, \varepsilon) \cap \mathbb{S}^{n}}\left(|u(y)|-\left|u_{\varepsilon}(x)\right|\right) d y \leq C f_{B(x, \varepsilon) \cap \mathbb{S}^{n}} f_{B(x, \varepsilon) \cap \mathbb{S}^{n}}|u(y)-u(z)| d y d z,
$$

and the last integral tends to 0 uniformly in $x$ as $\varepsilon \rightarrow 0$.

For further use, we note that the above argument implies that, if $u \in \operatorname{VMO}\left(\mathbb{S}^{n} ; F\right)$, where $F$ is any set, then

$$
\operatorname{dist}\left(u_{\varepsilon}(x), F\right) \rightarrow 0 \quad \text { uniformly in } x \text { as } \varepsilon \rightarrow 0 .
$$

Once (2.2) is proved, one may proceed as in the case of continuous maps.

Troubles come from Step 2. The following result essentially due to Brezis and Nirenberg [BNI].

Theorem 2.2. There is a degree (equivalently: the degree of smooth maps is continuous with respect to the $W^{s, p}$-norm) in $X$ if and only if $s p \geq n$.

Proof. When $s p>n$ or $s=n$ and $p=1$, we deal with continuous maps, so that we are done.

When $s p<n$, we may construct a sequence of smooth maps of degree 1 converging in $X$ to a constant; this implies that the degree of smooth maps does not pass to the limits. The construction is the following: fix a smooth map $v: \mathbb{R}^{n} \rightarrow \mathbb{S}^{n}$, of degree one and equal to a constant $P$ at infinity. Since $s p<n$, the scaled maps $v_{\varepsilon}(x)=v(x / \varepsilon)$ tend to $P$ in $W^{s, p}$ as $\varepsilon \rightarrow 0$. By stereographic projection, we may transport these maps on $\mathbb{S}^{n}$ and obtain a sequence of maps of degree 1 and converging to $P$ in $X$.

It remains to study the limiting case $s p=n$. Here, VMO comes again into the picture: maps in $\operatorname{VMO}\left(\mathbb{S}^{n} ; \mathbb{S}^{n}\right)$ do have a degree, i. e. the degree of smooth maps is continuous for BMO convergence. This is Theorem 1 in [BNI]. The argument there applies to the case where $\mathbb{S}^{n}$ is replaced by arbitrary compact oriented manifolds. We will present in the next section a different approach, tailored for $\mathbb{S}^{n}$-valued maps.

\subsection{Formulae for the degree of VMO maps}

In this part, maps $u$ are in $\operatorname{VMO}\left(\mathbb{S}^{n} ; \mathbb{S}^{n}\right)$. If $u$ is smooth, then there are two ways of computing its degree:

$$
\operatorname{deg} u=f_{\mathbb{S}^{n}} \operatorname{det}(D u, u)
$$


and

$$
\operatorname{deg} u=f_{B^{n+1}} \operatorname{Jac} v
$$

In the first formula, $u$ is considered as an $\mathbb{R}^{n+1}$-valued map, $D u$ stands for the tangential jacobian matrix and the determinant is $(n+1) \times(n+1)$. In the second one, $v: B^{n+1} \rightarrow \mathbb{R}^{n+1}$ is any Lipschitz extension of $u$, and Jac stands for the jacobian determinant.

It turns out that the second formula is "the right one", in the sense that we may use it to define the degree even if $u$ is merely VMO. In this case, one has to take a special extension $v$. The idea is borrowed from [BBM3]. Let $\tilde{u}$ be the harmonic extension of $u$ and set $v=\left\{\begin{array}{l}\tilde{u} /|\tilde{u}|, \text { if }|\tilde{u}| \leq 1 / 2 \\ 2 \tilde{u}, \text { if }|\tilde{u}| \leq 1 / 2\end{array}\right.$. When $u \in \mathrm{VMO}$, we have

$$
|\tilde{u}(x)| \rightarrow 1 \text { uniformly as }|x| \rightarrow 1
$$

This is proved in [BNII], Appendix 3. Though the rigorous proof is delicate, the result is intuitively clear: if $P_{r}$ is the Poisson kernel (so that $\tilde{u}=u * P_{r}$ ), then in some sense $P_{r}$ is close to a mollifier $\rho_{1-r}$, so that morally this result is similar to (2.2). This implies that the right-hand side of $(2.5)$ is well-defined. Indeed, near $\mathbb{S}^{n}, v$ is $\mathbb{S}^{n}$-valued, and thus its jacobian determinant vanishes. On the other hand, far away from $\mathbb{S}^{n}, v$ is Lipschitz. We may now try to define deg $u$ as the right-hand side of (2.5). Note that, when $u$ is smooth, $v$ is Lipschitz, so that we fall back to the classical degree. The following result completes the proof of Theorem 2.2 and incidentally gives, for $\mathbb{S}^{n}$-valued maps, an alternative proof of Theorem 1 in $[\mathrm{BNI}]$.

Proposition 2.1. The map $u \mapsto f_{B^{n+1}} \operatorname{Jac} v$ is continuous in $\operatorname{VMO}\left(\mathbb{S}^{n} ; \mathbb{S}^{n}\right)$.

Proof. Consider a sequence $\left\{u_{k}\right\}$ converging in VMO to some $u$. Then there is a fixed $r<1$ such that the corresponding harmonic extensions satisfy $\left|\widetilde{u_{k}}(x)\right| \geq 1 / 2$ and $|\tilde{u}(x)| \geq 1 / 2$ whenever $|x| \geq r$. To prove this, it suffices to check that the argument leading to (2.6) yields uniform estimates when applied to a convergent sequence. Thus, with obvious notations, we have

$$
\operatorname{deg} u_{k}=\frac{1}{\left|B^{n+1}\right|} \int_{B(0, r)} \operatorname{Jac} v_{k} \rightarrow \frac{1}{\left|B^{n+1}\right|} \int_{B(0, r)} \operatorname{Jac} v=\operatorname{deg} u
$$

since $\widetilde{u_{k}} \rightarrow \tilde{u}$ in $C^{1}(\bar{B}(0, r))$.

\subsection{Estimates for the degree}

Here, $X=W^{s, p}\left(\mathbb{S}^{n} ; \mathbb{S}^{n}\right)$ and $s p \geq n$ (so that the degree exists). A natural question is whether it is possible to estimate this degree in terms of the $W^{s, p}$-norm. Before giving the answer, let us consider the more familiar situation where maps are continuous or better. If $u$ is merely continuous, then there is no possible estimate, since the sup norm of $u$ is always 1 , while its degree can be any integer; the same argument shows that there is no estimate for the degree of VMO maps. However, if $u$ is slightly better, then there is a control; for example, if $u$ is Hölder continuous, then its degree is controlled by its Hölder semi-norm (this can be shown as in the proof of Theorem 2.3 below). In view of the Sobolev embeddings, we would thus expect the following: if $s p>n$, there is a control, while, if $s p=n$, there isn't. Surprisingly, the answer is [BBM3] 
Theorem 2.3. If $s p=n$, then

$$
|\operatorname{deg} u| \leq C|u|_{W^{s, p}}^{p} .
$$

Consequently, if $s p>n$ there is an estimate of the degree in terms of $|u|_{W^{s, p}}$.

Second assertion follows simply from the first one and Sobolev.

Proof. The case $s=n$ and $p=1$ is easy to treat (using (2.4)), so that we may assume $p>1$.

We start with a simple remark: if we know how to prove this result when $s$ is small, then we know how to prove it for all $s$. This follows from the Gagliardo-Nirenberg type embedding

$$
W^{s, p} \cap L^{\infty} \hookrightarrow W^{r, q} \quad \text { if } s p=r q \text { and } 0<r<s,
$$

valid except when $s$ is an integer, $p=1$ and $r>s-1$ is not an integer.

The case $p=1$ being settled, it thus suffices to treat the case $s<1$. We rely on (2.5). Let, for $x \in \mathbb{S}^{n}$, $r=r_{x} \in(0,1)$ be the smallest $\rho$ such that $|\tilde{u}(t x)|>1 / 2$ for $t \in(\rho, 1)$. Thus the set where the jacobian of $v$ does not vanish is contained in $U=\left\{\rho x ; x \in \mathbb{S}^{n}, \rho<r_{x}\right\}$. Since $\tilde{u}$ is the harmonic extension of a map of modulus 1, we have $|D \tilde{u}(y)| \leq C(1-|y|)^{-1}$ and thus $|\operatorname{Jac} v(y)| \leq C(1-|y|)^{-(n+1)}$. Integration of this inequality over $U$ yields $|\operatorname{deg} u| \leq C \int_{\mathbb{S}^{n}}\left(1-r_{x}\right)^{-n} d x$. Next, with $I_{x}$ denoting the segment connecting $x$ to $r_{x} x$, we have either $r_{x}=0$ or

$$
1 / 2 \leq\left|u(x)-\tilde{u}\left(r_{x} x\right)\right| \leq\left(1-r_{x}\right)^{n / p}|\tilde{u}|_{C^{n / p}\left(I_{x}\right)} \leq C\left(1-r_{x}\right)^{n / p}|\tilde{u}|_{W^{(n+1) / p, p}\left(I_{x}\right)} .
$$

Thus

$$
\left(1-r_{x}\right)^{-n} \leq C|\tilde{u}|_{W^{(n+1) / p, p\left(I_{x}\right)}}^{p}+1 .
$$

Integrating this inequality yields, with the help of trace theory, that $|\operatorname{deg} u| \leq C|u|_{W^{s, p}}^{p}+C^{\prime}$. Finally, it is easy to get rid of $C^{\prime}$ by noting that, when the semi-norm of $u$ is small, $u$ is close in VMO to a constant, and thus its degree vanishes.

There is a challenging question concerning estimate (2.7). We state it for $\mathbb{S}^{1}$, though it also makes sense for $\mathbb{S}^{n}$.

Open Problem 1. Let $1<p<\infty$. Set

$$
C=\inf \left\{I=\int_{\mathbb{S}^{1}} \int_{\mathbb{S}^{1}} \frac{|u(x)-u(y)|^{p}}{|x-y|^{2}} d x d y=|u|_{W^{1 / p, p}\left(\mathbb{S}^{1}\right)}^{p} ; u: \mathbb{S}^{1} \rightarrow \mathbb{S}^{1}, \operatorname{deg} u=1\right\} .
$$

\section{Is $C$ attained? Which are the optimal functions?}

Note that $C>0$. The answer is easy to give when $p=2$ (and unknown when $p \neq 2$ ). Indeed, when $p=2$ and $u=\sum a_{n} e^{\imath n \theta}$, an easy computation yields $I=4 \pi^{2} \sum|n|\left|a_{n}\right|^{2}$, while deg $u=\sum n\left|a_{n}\right|^{2}$ (see Section 2.4). Thus $I \geq 4 \pi^{2}$. Equality requires $a_{n}=0$ when $n<0$. Thus $u$ is the boundary value of a holomorphic map. It is easy to see that the holomorphic maps in the unit disk that are of modulus 1 and degree 1 on the unit circle are precisely the Moebius transforms. Thus $C=4 \pi^{2}$ and optimal maps are Moebius transforms.

Note that this problem lacks of compactness, which explains why it may be difficult to handle: if $\left\{u_{k}\right\}$ is a minimizing sequence, then up to some subsequence $\left\{u_{k}\right\}$ converges weakly in $W^{1 / p, p}$ to some $u$. However, weak convergence does not guarantees that the degree of $u$ is 1 (take, e. g., $u_{k}\left(e^{\imath \theta}\right)=\left\{\begin{array}{ll}e^{\imath k \theta}, & \text { if } 0 \leq \theta \leq 1 / k \\ 1, & \text { otherwise }\end{array}\right.$, which has degree 1 and weakly converges to the constant 1$)$. Thus it is unclear whether the infimum is attained. 


\subsection{Maps from $\mathbb{S}^{1}$ into $\mathbb{S}^{1}$ : an explicit degree formula}

Throughout this section, $u: \mathbb{S}^{1} \rightarrow \mathbb{S}^{1}$. If $u$ is smooth and $u=\sum a_{n} e^{\imath n \theta}$, then (2.5) reads

$$
\operatorname{deg} u=\sum n\left|a_{n}\right|^{2}
$$

this was first noted by Brezis. On the other hand, as we saw right after Open Problem 1, $|u|_{H^{1 / 2}}^{2} \sim$ $\sum|n|\left|a_{n}\right|^{2}$. Using this remark and the continuity of the degree in $H^{1 / 2}$ (Theorem 2.2), we find

Proposition 2.2. Degree formula (2.10) is valid when $u \in H^{1 / 2}\left(\mathbb{S}^{1} ; \mathbb{S}^{1}\right)$.

Consequently, (2.10) holds if $u \in W^{1 / p, p}$ for $1<p<2$.

Last statement is simply a consequence of the Gagliardo-Nirenberg inequality.

In a survey paper [B1], Brezis asked several challenging questions about formula (2.10). Question 1: since degree makes sense when $u$ is merely continuous (or even VMO), can one give a meaning to the right-hand side of (2.10) in order to recover deg $u$ from $\left|a_{n}\right|$ ? Soon after, this answer revealed to be (presumably) negative (Korevaar $[\mathrm{Ko}]$ ). This suggested some more "modest" questions. Question 2: if $u=\sum a_{n} e^{\imath n \theta}, v=\sum b_{n} e^{\imath n \theta}$ are continuous maps such that $\left|a_{n}\right|=\left|b_{n}\right|$, is it true that $\operatorname{deg} u=\operatorname{deg}$ $v$ ? Answer: no (Bourgain and Kozma). Question 3: same as Question 2 if $u, v \in W^{1 / p, p}$ and $p>2$. This is partially open.

Back to Question 1: the absolute convergence of the series $\sum n\left|a_{n}\right|^{2}$ is equivalent to $u \in H^{1 / 2}$. Nevertheless, one may still hope give a meaning to its sum. Commonly used summation procedures consist in taking either $S=\lim _{k \rightarrow \infty} \sum_{-k}^{k} n\left|a_{n}\right|^{2}$ or $T=\lim _{r \rightarrow 1-} \sum r^{|n|} n\left|a_{n}\right|^{2}$. Korevaar's result concerns these two procedures.

Theorem 2.4. If $u \in C^{0}\left(\mathbb{S}^{1} ; \mathbb{S}^{1}\right)$, then $S$ or $T$ could be any real number, or even not exist.

The proof is explicit: given $\alpha \in \mathbb{R}$, Korevaar exhibits a map $u$ such that $S=\alpha$ (or $T=\alpha$ ). Of course, one may imagine some other summation procedure, but Korevaar's construction will probably take care of it.

Concerning Question 3, the first answer was obtained for Hölder maps in an unpublished work of Kahane. In the setting of Sobolev spaces, Brezis [B2] proved the following variant of Kahane's result.

Theorem 2.5. If $u \in W^{1 / 3,3}$, then

$$
\operatorname{deg} u=\lim _{\varepsilon \rightarrow 0} \sum_{n \neq 0} \frac{\sin ^{2} n \varepsilon}{n \varepsilon^{2}}\left|a_{n}\right|^{2}
$$

Consequently, the answer to Question 3 is positive when $p \leq 3$.

Proof. We assume $u$ continuous; the general case requires some more subtle consideration on lifting, developed in Section 3. Write $u=z^{d} e^{\imath \psi}$, where $d=\operatorname{deg} u$ and $\psi$ is continuous. It is easy to see that $\psi \in W^{1 / 3,3}$. The starting point is the identity

$$
\operatorname{Im} \int_{0}^{2 \pi} u\left(e^{\imath(\theta+h)}\right) \bar{u}\left(e^{\imath \theta}\right) d \theta=2 \pi \sum\left|a_{n}\right|^{2} \sin n h=\int_{0}^{2 \pi} \sin \left(d h+\psi\left(e^{\imath(\theta+h)}\right)-\psi\left(e^{\imath \theta}\right)\right) d \theta .
$$

A second order Taylor expansion of $\sin \left(d h+\psi\left(e^{\imath(\theta+h)}\right)-\psi\left(e^{\imath \theta}\right)\right)$ yields

$$
\left.\left|\sum\right| a_{n}\right|^{2} \sin n h-\left.d h|\leq C| h\right|^{2}+C \int_{0}^{2 \pi}\left|\psi\left(e^{\imath(\theta+h)}\right)-\psi\left(e^{\imath \theta}\right)\right|^{3} d \theta .
$$


Integrating this inequality over $h \in(0,2 \varepsilon)$ and dividing the result by $2 \varepsilon^{2}$ lead to

$$
\left.\left|\sum_{n \neq 0} \frac{\sin ^{2} n \varepsilon}{n \varepsilon^{2}}\right| a_{n}\right|^{2}-d\left|\leq C \varepsilon+\frac{C}{\varepsilon^{2}} \int_{0}^{2 \varepsilon} \int_{0}^{2 \pi}\right| \psi\left(e^{\imath(\theta+h)}\right)-\left.\psi\left(e^{\imath \theta}\right)\right|^{3} d \theta d h .
$$

Using the fact that $\psi \in W^{1 / 3,3}$, it is easy to see that the right-hand side of the above inequality tends to 0 with $\varepsilon$.

In some sense, the above result is optimal: Kahane [Ka] proved that, if $u \in W^{1 / p, p}$ for some $p>3$, then the limit in (2.11) may be any real number. However, this still leaves the following

Open Problem 2. For $p>3$, can one compute the degree of $u=\sum a_{n} e^{\imath n \theta} \in W^{1 / p, p}$ in terms of $\left|a_{n}\right|$ ?

\subsection{Another degree}

As we saw in Sections 2.1-2.2, one may prove existence of a degree first by establishing density of smooth maps, next by using (2.5). Yet there is another natural way to do it: assume that the integrals in (2.4) or (2.5) make sense, take this as the definition of the degree, and then prove that the result is an integer. This approach was taken by Esteban and Müller [EM]. In what follows, it is convenient to consider $u$ not as a map from $\mathbb{S}^{n}$ into $\mathbb{R}^{n+1}$, but rather as an $\mathbb{S}^{n}$-valued map. With this in mind, the jacobian matrix of $u$ is $n \times n$, and (2.4) rewrites

$$
\operatorname{deg} u=f_{\mathbb{S}^{n}} \operatorname{Jac} u
$$

Theorem 2.6. Assume that $u \in W^{1, n-1}\left(\mathbb{S}^{n} ; \mathbb{S}^{n}\right)$ is such that all the $(n-1) \times(n-1)$ minors of its jacobian matrix are in $L^{n /(n-1)}$. Then the right-hand side of (2.12) is an integer.

Note that the hypotheses imply that Jac $u \in L^{1}$. The argument relies essentially on the area formula of Federer: if Jac $u \in L^{1}$, then there is an integer-valued $L^{1}$-function $d$ on $\mathbb{S}^{n}$ such that

$\int f \circ u(x) \operatorname{Jac} u(x) d x=\int f(y) d(y) d y$ whenever $f: \mathbb{S}^{n} \rightarrow \mathbb{R}$ is smooth. The theorem amounts then to proving that $d$ is constant.

The VMO degree and the degree defined in the above theorem are not related: if $u \in$ VMO, we need not have $u \in W^{1, n-1}$. Conversely, a map that satisfies the assumptions of the theorem need not belong to VMO: pick a map $\psi \in W^{1,1}\left(\mathbb{S}^{2} ;(-1 / 2,1 / 2)\right)$ which does not belong to VMO, and set $u=\left(\psi, \sqrt{1-\psi^{2}}, 0\right)$, which is $\mathbb{S}^{2}$-valued. Then the first order minors of $u$ are in $L^{1}$, since $\psi \in W^{1,1}$, while its jacobian determinant vanishes, since $u$ is $\mathbb{S}^{1}$-valued. Clearly, $u$ does not belong to VMO. This leaves us with the following very vague question.

Open Problem 3. Is there a "unified" degree theory?

The above theorem was generalized by Giaquinta, Modica and Souček [GMS1]. We do not quote here their result, which needs notions of cartesian currents to be stated.

\subsection{Degree beyond VMO}

Let $u$ be a map from $\mathbb{S}^{n} \times(0,1)^{k}$ into $\mathbb{S}^{n}$. If $u$ is continuous, then one may define a degree of $u$ as follows: fix any $\lambda \in(0,1)^{k}$ and set $\operatorname{deg} u=\operatorname{deg} u(\cdot, \lambda)$. By homotopical invariance of the degree, this definition does not depend on $\lambda$ and yields a degree which is continuous for the sup norm. The same 
can be done if $u \in W^{s, p}$, with $s p \geq n+k$. Indeed, by Gagliardo-Nirenberg we may assume $s<1$. By trace theory, $\lambda \mapsto u(\cdot, \lambda)$ is continuous from $(0,1)^{k}$ into $W^{s-k / p, p}$, and thus into VMO. The degree being continuous for the BMO norm, we derive that $\operatorname{deg} u(\cdot, \lambda)$ does not depend on $\lambda$. Thus we may define a degree in $W^{s, p}$ if $W^{s, p}$ embeds into VMO (which is the same as $s p \geq n+k$ ). It turns out that the condition $s p \geq n+k$ can be relaxed. In special cases, the following result was obtained by White [W]; see also Rubinstein and Sternberg [RS]. The general case is taken from [BLMN].

Theorem 2.7. Assume that $s p \geq n+1$. Let $u \in W^{s, p}\left(\mathbb{S}^{n} \times(0,1)^{k} ; \mathbb{S}^{n}\right)$. Then there is an integer $d$ such that $\operatorname{deg} u(\cdot, \lambda)=d$ for a. e. $\lambda \in(0,1)^{k}$.

Thus, we may define the degree of $u$ as this integer.

In addition, the condition $s p \geq n+1$ is optimal and the degree is continuous for the $W^{s, p}$-norm.

Proof. The case $k=1$ is settled by the discussion at the beginning at this section. Assume thus $k \geq 2$.

Note that, for a. e. $\lambda, u(\cdot, \lambda) \in W^{s, p} \subset \mathrm{VMO}$, so that the map $\psi$ given by $\psi(\lambda)=\operatorname{deg} u(\cdot, \lambda)$ is defined a. e. With some work, one may prove that $\psi$ is measurable. For a. e. $t_{1}, \ldots, t_{i-1}, t_{i+1}, \ldots t_{k} \in(0,1)$, the map $u\left(\cdot, t_{1}, \ldots, t_{i-1}, \cdot, t_{i+1}, \ldots t_{k}\right)$ is in $W^{s, p}$. If this is the case, then $\psi\left(t_{1}, \ldots, t_{i-1}, \cdot, t_{i+1}, \ldots t_{k}\right)$ is constant a. e. (cf the case $k=1$ ). Existence of the degree follows from the following elementary

Lemma 2.1. Let $\psi:(0,1)^{k} \rightarrow \mathbb{R}$ be a measurable function such that for a. e. $t_{1}, \ldots, t_{i-1}, t_{i+1}, \ldots t_{k} \in$ $(0,1)$, the map $\psi\left(t_{1}, \ldots, t_{i-1}, \cdot, t_{i+1}, \ldots t_{k}\right)$ is constant a. e. Then $\psi$ is constant $a$. $e$.

To prove continuity of degree, let $u_{k} \rightarrow u$ in $W^{s, p}$. Possibly after passing to a subsequence, we then have $u_{k}(\cdot, \lambda) \rightarrow u(\cdot, \lambda)$ in $W^{s, p}$ for a. e. $\lambda$. We conclude using continuity of the degree in VMO. Incidentally, this proves continuity for the $W^{r, q}$-norm as soon as $r q \geq n$.

To prove optimality, let $k=1, e \in \mathbb{S}^{n}$ and $u(x, \lambda)=(x-2 \lambda e) /|x-2 \lambda e|$. Then $u \in W^{s, p}$ if $s p<n+1$, while $\operatorname{deg} u(\cdot, \lambda)=\left\{\begin{array}{ll}1, & \text { if } \lambda<1 / 2 \\ 0, & \text { if } \lambda>1 / 2\end{array}\right.$.

\subsection{General manifolds}

One may define a degree for VMO maps from $M$ into $N$, provided these manifolds have same dimension, are compact, oriented and without boundary. Density of smooth maps in $\operatorname{VMO}(M ; N)$ follows from (2.3). Existence of degree was proved by Brezis and Nirenberg [BNI].

Theorem 2.8. The map $C^{\infty}(M ; N) \ni u \mapsto \operatorname{deg} u$ is continuous for the BMO norm.

Their proof does not use integral formulae for the degree; yet, this can probably be done this way. The above result allows to state, e. g., Theorem 2.7 for maps from $M \times(0,1)^{k}$ into $N$. It is also plausible that Theorems 2.3 and 2.6 are still valid for maps from $M$ into $N$.

For a map $u$ from $\mathbb{S}^{n}$ into itself, one of the interests of the degree is that it describes the homotopy class of $u$ : Hopf's theorem asserts that if two continuous maps have the same degree, then they are homotopic; this holds also for VMO [BNI]. In general, it is more natural to replace the degree with the homotopy class. This can be done indeed: one can associate to VMO maps a homotopy class, which is continuous with respect to BMO convergence (this is obtained by copying the proof of Theorem 1 in $[\mathrm{BNI}])$. By mimicking the proof of Theorem 2.7, one may prove the following result, essentially due to White $[\mathrm{W}]$ (see also [HaL2]):

Theorem 2.9. Let $u \in W^{s, p}\left(M \times(0,1)^{k} ; N\right)$, with $M, N$ compact (but not necessarily of the same dimension) and $s p \geq \operatorname{dim} M+1$. Let, for $\lambda \in(0,1)^{k}, d(\lambda)$ be the homotopy class of $u(\cdot, \lambda)$ in $C(M ; N)$ (this is well-defined when $u(\cdot, \lambda) \in W^{s, p}$, thus a. e.). Then $d$ is constant a. e. 
We may thus define in this way $[u]$, the homotopy class of $u$.

In addition, $u \mapsto[u]$ is continuous for the $W^{s, p}$-convergence.

\section{$3 \quad \mathbb{S}^{1}$-valued maps: lifting}

The question we address here is: given an $\mathbb{S}^{1}$-valued map $u$, can one find a lifting of $u$ as smooth as $u$ ? In the well-known case of continuous maps on domains in $\mathbb{R}^{n}$, the answer is positive locally (i. e. on balls), while globally the answer may be negative, due to the topology of the domain. In the context of Sobolev spaces, the local problem is already interesting. We assume throughout this section that $u: C \rightarrow \mathbb{S}^{1}$, where $C$ is the unit cube in $\mathbb{R}^{n}$.

\subsection{Lifting of Sobolev maps}

Here, $u \in W^{s, p}$, and we look for a real function $\varphi \in W^{s, p}$ such that $u=e^{\imath \varphi}$. We start with the question of the uniqueness: if $\varphi_{1}, \varphi_{2} \in W^{s, p}$ lift $u$, is it true that $\varphi_{1}-\varphi_{2}$ is a constant multiple of $2 \pi$ ? This amounts to proving that, if $\varphi: C \rightarrow \mathbb{Z}$ is in $W^{s, p}$, then $\varphi$ is constant.

Proposition 3.1. The only functions in $W^{s, p}(C ; \mathbb{Z})$ are constants if and only if $s p \geq 1$.

In special cases, this was proved by Hardt, Kinderlehrer and Lin [HKL2] and Bethuel and Demengel [BD]. The general case is from [BLMN].

Proof. Let $Q \varsubsetneqq B$ be a cube. If $s p<1$, then the characteristic function of $Q$ belongs to $W^{s, p}(C ; \mathbb{Z})$; this proves the necessity of the condition $s p \geq 1$.

Suppose now $s p \geq 1$. Fix some $i \in\{1, \ldots, n\}$. For almost every $x_{j} \in(0,1), j \neq i$, the map $v$ given by $v(t)=u\left(x_{1}, \ldots, x_{i-1}, t, x_{i+1}, \ldots, x_{n}\right)$ belongs to $W^{s, p}((0,1) ; \mathbb{Z})$. If we mollify $v,(2.3)$ implies that the maps $v_{\varepsilon}$ are close to $\mathbb{Z}$; since these maps are smooth, they have to be close to a fixed integer when $\varepsilon$ is small. Passing to the limits, we find that $v$ is constant a.e. Lemma 2.1 implies that $u$ is constant a. e.

We next give an example of map with no lifting. Let $u: B^{2} \rightarrow \mathbb{S}^{1}, u(z)=z /|z|$, which belongs to $W^{1,1}$. We claim that $u$ has no lifting in $W^{1,1}$. Argue by contradiction: $u=e^{\imath \varphi}$, with $\varphi \in W^{1,1}$. For a. e. $r \in(0,1), \varphi$ restricted to the circle $C(0, r)$ has a continuous representative, still denoted $\varphi$, such that $u=e^{\imath \varphi}$ everywhere on $C(0, r)$. This is impossible, since on any such circle the winding number of $u$ is 1 , while the one of $e^{\imath \varphi}$ is 0 .

In general, the question of existence of $\varphi$ was settled in [BBM1].

Theorem 3.1. There is a lifting $\varphi \in W^{s, p}$ for each $u \in W^{s, p}$ except when:

a) $n \geq 2$ and $s p \in[1,2)$

or

b) $n \geq 2, s<1$ and $s p \in[2, n)$.

Proof. Several cases are to be considered:

(i) When $s p>n, u$ is continuous. We then take $\varphi$ to be any continuous lifting of $u$; it is easy to see that $\varphi \in W^{s, p}$.

(ii) When $s \geq 1$ and $s p \geq 2$, the idea of the proof goes back to a paper of Carbou [C]. Assume that $\varphi$ exists. Since $u=e^{\imath \varphi}$, we find that $D u=\imath u D \varphi$, so that $D \varphi=F$, with $F=-\imath \bar{u} D u$; here it is where the hypothesis $s \geq 1$ plays a role. The idea is then to solve the equation $D \varphi=F$, and to prove that the solution is essentially the one needed. One may prove that $F \in W^{s-1, p}$; this relies 
on multiplication properties of Sobolev spaces. On the other hand, $F$ is a closed vector field, i. e., $\partial F_{i} / \partial x_{j}=\partial F_{j} / \partial x_{i}$. Formally, this is clear: if $u=u_{1}+\imath u_{2}$, then $F=u_{1} D u_{2}-u_{2} D u_{1}$. Therefore (at least if $u$ is smooth)

$$
\partial F_{i} / \partial x_{j}-\partial F_{j} / \partial x_{i}=2\left(\partial u_{1} / \partial x_{j} \partial u_{2} / \partial x_{i}-\partial u_{1} / \partial x_{i} \partial u_{2} / \partial x_{j}\right)
$$

The rigorous justification of this equality is obtained by approximating $u$ with smooth maps and requires $s p \geq 2$. Next the right-hand side of (3.1) vanishes. Indeed, $u$ is $\mathbb{S}^{1}$-valued, so that $D u_{1}$ and $D u_{2}$ are collinear.

A variant of Poincaré's lemma allows then to write $F=D \psi$ for some $\psi \in W^{s, p}$. Finally, the map $u e^{-\imath \psi}$ is constant (it is easy to check that its gradient vanishes), so that $\varphi=\psi+C$, with $C$ appropriate constant, is a lifting of $u$.

(iii) The case $s p=n$. In order to keep the presentation simple, we consider the special case of $H^{1 / 2}$ maps on an interval; the general case follows the same lines. We regularize $u$ : set $v(x, \varepsilon)=u * \rho_{\varepsilon}(x)$. By trace theory, $v \in H^{1}\left((0,1)^{2}\right)$. Since $u \in \mathrm{VMO}, v$ has modulus close to 1 for small $\varepsilon$. Thus $w=v /|v|$ is $H^{1}$ and of modulus 1 in $(0,1) \times(0, \delta)$ for small $\delta$. In view of case (ii), we may write $w=e^{\imath \psi}$, with $\psi \in H^{1}$. By taking traces, we have $u=e^{\imath \varphi}$, with $\varphi=\operatorname{tr} \psi \in H^{1 / 2}$.

(iv) Cases a) or b). Explicit examples show non existence.

(v) The case $s p<1$. It is the delicate one, and we refer to [BBM1] for details.

\subsection{Estimates for the lifting}

Once existence of lifting is established, the natural question is whether we may estimate $|\varphi|_{W^{s, p}}$ in terms of $|u|_{W^{s, p}}$. The proof of the above theorem is constructive, and yields estimates except when we are in the critical case $s p=n$. Actually, in this case there is no estimate. Here is an example. Let $n=1($ so that $C=(0,1))$ and let $1<p<\infty$. Let $\varphi_{k}(x)= \begin{cases}0, & \text { if } 0<x<1 / 2 \\ 2 k \pi(x-1 / 2), & \text { if } 1 / 2<x<1 / 2+1 / k \\ 2 \pi, & \text { otherwise }\end{cases}$ and set $u_{k}=e^{\imath \varphi_{k}}$. It is easy to see that $\left\{u_{k}\right\}$ is bounded in $W^{1 / p, p}$. Since $\varphi_{k}$ belongs to $W^{1 / p, p}$, any lifting of $u_{k}$ in $W^{1 / p, p}$ is $\varphi_{k}+C$, by Proposition 3.1. It is easy to see that $\left\{\left|\varphi_{k}\right|_{W^{1 / p, p}}\right\}$ is not bounded. Thus, in this limiting case there is no control of $\varphi$ in terms of $u$.

However, the above $\varphi_{k}$ 's are bounded in $W^{1,1}$. This suggests that there is a control of the phase, if not in $W^{1 / p, p}$, then in a space containing $W^{1 / p, p}$ and $W^{1,1}$. For some values of $p$, this was proved by Bourgain and Brezis [BB].

Theorem 3.2. Let $1<p \leq 2$. Then each $u \in W^{1 / p, p}\left((0,1) ; \mathbb{S}^{1}\right)$ may be written as

$$
u=e^{\imath\left(\varphi_{1}+\varphi_{2}\right)}, \quad \text { where }\left|\varphi_{1}\right|_{W^{1,1}} \leq C|u|_{W^{1 / p, p}}^{p} \text { and }\left|\varphi_{2}\right|_{W^{1 / p, p}} \leq C|u|_{W^{1 / p, p}} .
$$

More generally, one may replace $(0,1)$ by $(0,1)^{n}$, but then $u$ has to be in the closure of $\mathbb{S}^{1}$-valued smooth maps.

Their result is stated only for $p=2$, but the proof works also when $1<p<2$.

Proof. The construction in [BB] is explicit, but not elementary; it relies on a Littlewood-Paley decomposition of $u$. In one dimension, it is easy to establish a weaker form of (3.2), namely $u=e^{\imath \varphi}$, with

$$
|\varphi|_{W^{1,1}+W^{1 / p, p}} \leq C\left(|u|_{W^{1 / p, p}}+|u|_{W^{1 / p, p}}^{p}\right)
$$


For simplicity, we work only with $p=2$ and $u \in C^{\infty}$, though this is not relevant. Let $\varphi$ be any smooth lifting of $u$. It suffices to prove that $\left|\varphi^{\prime}\right|_{L^{1}+H^{-1 / 2}} \leq C\left(|u|_{H^{1 / 2}}+|u|_{H^{1 / 2}}^{2}\right)$. By duality, this amounts to proving

$$
\left|\int_{0}^{1} \varphi^{\prime} \zeta\right| \leq C\left(|u|_{H^{1 / 2}}+|u|_{H^{1 / 2}}^{2}\right)\left(\|\zeta\|_{L^{\infty}}+|\zeta|_{H^{1 / 2}}\right), \quad \forall \zeta \in C_{0}^{\infty}(0,1) .
$$

Using the identity $\varphi^{\prime}=-\imath \bar{u} u^{\prime}$, integration by parts shows that

$$
\left|\int_{0}^{1} \varphi^{\prime} \zeta\right|=\left|\int_{0}^{1} u^{\prime}(\bar{u} \zeta)\right| \leq\left|u^{\prime}\right|_{H^{-1 / 2}}|\bar{u} \zeta|_{H^{1 / 2}}=|u|_{H^{1 / 2}}|\bar{u} \zeta|_{H^{1 / 2}}
$$

and (3.4) follows from the elementary inequality $|\bar{u} \zeta|_{H^{1 / 2}} \leq|u|_{H^{1 / 2}}\|\zeta\|_{L^{\infty}}+|\zeta|_{H^{1 / 2}}$.

Actually, one can carry out this computation in any dimension, and find an estimate for $D \varphi$ in $L^{1}+H^{-1 / 2}$. However, in $n \geq 2$ dimensions, it is unclear whether $D \varphi \in L^{1}+H^{-1 / 2}$ implies $\varphi \in$ $W^{1,1}+H^{1 / 2}$ !

Open Problem 4. Is it true that Theorem 3.2 is still valid for $p>2$ ?

This is not known even in one dimension. An estimate weaker then (3.2) was established in [BBM3], Theorem 0.1.

\subsection{Lifting of VMO maps}

This was settled in [BNI].

Theorem 3.3. Each $u \in \operatorname{VMO}\left(C ; \mathbb{S}^{1}\right)$ has a lifting $\varphi \in \mathrm{VMO}$, unique modulo constants.

Proof. Uniqueness comes from the fact that integer-valued VMO maps are constant, cf proof of Proposition 3.1. Concerning existence, the idea is to regularize $u$. For small $\varepsilon$, say $\varepsilon<\varepsilon_{0}$, the maps $v_{\varepsilon}=u_{\varepsilon} /\left|u_{\varepsilon}\right|$ are well-defined, of modulus 1, and depending continuously on $\varepsilon$. Thus we may write $v_{\varepsilon}=e^{\imath \varphi_{\varepsilon}}$, where $\varphi_{\varepsilon}$ is continuous and depends continuously on $\varepsilon$. For a.e. $x$, we have $\lim _{\varepsilon \rightarrow 0} e^{\imath \varphi_{\varepsilon}(x)}=u(x)$; this implies that $\varphi(x)=\lim _{\varepsilon \rightarrow 0} \varphi_{\varepsilon}(x)$ exists a. e. and satisfies $u=e^{\imath \varphi}$. It remains to check that $\varphi \in \mathrm{VMO}$, and the idea is to prove that the family $\left\{\varphi_{\varepsilon}\right\}$ is relatively compact in VMO. To explain how this is obtained, we replace for simplicity $C$ by $\mathbb{S}^{n}$. With the notations in $(2.1)$, set $M_{\delta}(u)=\sup _{\lambda \leq \delta} I_{\delta}(u)$. The necessary and sufficient condition for relatively compactness in VMO is $\lim _{\delta \rightarrow 0} \sup _{\varepsilon<\varepsilon_{0}} M_{\delta}\left(\varphi_{\varepsilon}\right)=0$; see [BNI]. Now Taylor's formula yields

$$
\left|\varphi_{\varepsilon}(y)-\varphi_{\varepsilon}(y)\right| \leq\left|v_{\varepsilon}(y)-v_{\varepsilon}(z)\right|+1 / 2\left|\varphi_{\varepsilon}(y)-\varphi_{\varepsilon}(y)\right|^{2} .
$$

Thus

$$
M_{\delta}\left(\varphi_{\varepsilon}\right) \leq M_{\delta}\left(v_{\varepsilon}\right)+1 / 2 \sup _{\lambda \leq \delta} \sup _{x \in \mathbb{S}^{n}} f_{B(x, \lambda) \cap \mathbb{S}^{n}} f_{B(x, \lambda) \cap \mathbb{S}^{n}}\left|\varphi_{\varepsilon}(y)-\varphi_{\varepsilon}(z)\right|^{2} d y d z,
$$

and the John-Nirenberg inequality implies that the last quantity in the above formula is less than $C M_{\delta}^{2}\left(\varphi_{\varepsilon}\right)$. This leads to

$$
M_{\delta}\left(\varphi_{\varepsilon}\right) \leq M_{\delta}\left(v_{\varepsilon}\right)+C M_{\delta}^{2}\left(\varphi_{\varepsilon}\right)
$$

This implies easily that, for small $\delta$, we have $M_{\delta}\left(\varphi_{\varepsilon}\right) \leq C M_{\delta}\left(v_{\varepsilon}\right)$. Compactness follows then from the fact that the last supremum tends to 0 with $\delta$, uniformly in $\varepsilon$, since $\left\{v_{\varepsilon}\right\}$ is compact in VMO. 
A similar argument gives a proof (different from the original one) of the following result of Coifman and Meyer [CM].

Theorem 3.4. There is some $\delta>0$ such that, if $u \in \operatorname{BMO}\left(C ; \mathbb{S}^{1}\right)$ and $|u|_{\mathrm{BMO}} \leq \delta$, then $u=e^{\imath \varphi}$, with $|\varphi|_{\mathrm{BMO}} \leq C|u|_{\mathrm{BMO}}$

Note that a BMO map always has a BMO lifting: for example, its main argument is in BMO. The point in this theorem is that, if $u$ is close (in BMO) to a constant, then the phase $\varphi$ is controlled in BMO by $u$.

\subsection{Lifting in BV}

When $u \in \mathrm{BV}\left(\Omega ; \mathbb{S}^{1}\right), u$ has a lifting in BV (Giaquinta, Modica and Souček [GMS2]); here, $\Omega$ could be any smooth domain or manifold. This result was sharpened by Dávila and Ignat [DI].

Theorem 3.5. For $u \in \mathrm{BV}\left(\Omega ; \mathbb{S}^{1}\right)$, there is some $\varphi \in \mathrm{BV}$ such that $u=e^{\imath \varphi}$ and

$$
|\varphi|_{\mathrm{BV}} \leq 2|u|_{\mathrm{BV}}
$$

The constant 2 is optimal.

Proof. The constant 2 cannot be improved. Indeed, let $u$ be the identity on $\mathbb{S}^{1}$ and let $\varphi$ be a BV lifting of $u$. Then $\varphi$ has to jump somewhere, say at 1 . Since $u=e^{\imath \varphi}$ a. e., this implies that $|\varphi(1+)-\varphi(1-)| \geq 2 \pi$. On the other hand, the variation of $\varphi$ on $\mathbb{S}^{1} \backslash\{1\}$ is at least $\int_{\mathbb{S}^{1}}|D u|=2 \pi$. Consequently, the total variation of $\varphi$ is at least $4 \pi$, while the one of $u$ is $2 \pi$.

Concerning (3.5), we give a proof when $u$ is smooth; this case is of interest only when $\Omega$ is not simply connected, for otherwise we may write $u=e^{\imath \varphi}$ with $\varphi$ smooth and $|D \varphi|=|D u|$. Let arg be the principal argument (which has a jump at $\pi$ ) and set, for $\alpha \in[0,2 \pi), \theta_{\alpha}(z)=\arg \left(z e^{\imath(\pi-\alpha)}\right)$, which has a jump at $\alpha$. If $e^{\imath \alpha}$ is a regular value of $u$, then $\varphi_{\alpha}=\theta_{\alpha}(u)$ jumps by $2 \pi$ on the smooth level set $L_{\alpha}=u^{-1}\left(e^{\imath \alpha}\right)$. Outside this set, $\varphi_{\alpha}$ is smooth and $\left|D \varphi_{\alpha}\right|=|D u|$. Thus,

$$
\left|\varphi_{\alpha}\right|_{\mathrm{BV}}=2 \pi\left|L_{\alpha}\right|+|u|_{\mathrm{BV}}
$$

where the measure of $L_{\alpha}$ is the $(n-1)$-dimensional Hausdorff measure. The idea is that, for some $\alpha$, $\varphi_{\alpha}$ will satisfy (3.5). To see, this, we integrate (3.6) over $\alpha \in[0,2 \pi)$ and find, with the help of the co-area formula, that

$$
\int_{0}^{2 \pi}\left|\varphi_{\alpha}\right|_{\mathrm{BV}} d \alpha=2 \pi \int_{0}^{2 \pi}\left|L_{\alpha}\right| d \alpha+2 \pi|u|_{\mathrm{BV}}=4 \pi|u|_{\mathrm{BV}}
$$

This gives immediately the existence of an appropriate $\alpha$.

The heart of the proof in $[\mathrm{DI}]$ is to establish the inequality $\int_{0}^{2 \pi}\left|\varphi_{\alpha}\right|_{\mathrm{BV}} d \alpha \leq 4 \pi|u|_{\mathrm{BV}}$ when $u$ is merely $\mathrm{BV}$; the argument relies heavily on the structure of BV maps.

\subsection{Lifting when there is no lifting}

Throughout this section, $n \geq 2$. If $u \in W^{1,1}\left(C ; \mathbb{S}^{1}\right)$, then $u$ may not have a lifting in $W^{1,1}$, by Theorem 3.1. However, by Theorem $3.5 u$ has a lifting in BV, which is a slightly larger space. Another example in the same vein is [BBM2] 
Theorem 3.6. Each $u \in H^{1 / 2}\left((0,1)^{2} ; \mathbb{S}^{1}\right)$ has a lifting in $H^{1 / 2}+\mathrm{BV}$.

Using Theorem 3.2 and results form Alberti, Baldo and Orlandi [ABO], one could more generally prove that maps in $W^{1 / p, p}\left((0,1)^{n} ; \mathbb{S}^{1}\right)$ have a lifting in in $W^{1 / p, p}+\mathrm{BV}$ when $1<p \leq 2$. However, this is not known when $p>2$.

Open Problem 5. Is it true that, for each $s, p$ and $n$, a map in $W^{s, p}\left((0,1)^{n} ; \mathbb{S}^{1}\right)$ has a lifting in $W^{s, p}+\mathrm{BV}$ ?

\subsection{Lifting in covering spaces}

One could envision, more generally, maps $u$ from $C$ into some smooth compact manifold $N$. If $Z$ is a universal cover of $N$ and $\pi$ is the projection of $Z$ onto $N$, a natural question is whether we may write $u=\pi \circ \varphi$, with $\varphi$ as smooth as $u$. In VMO, this was answered by Brezis and Nirenberg [BNI].

Theorem 3.7. Each $u \in \operatorname{VMO}(C ; N)$ may be written as $u=\pi \circ \varphi$, with $\varphi \in \operatorname{VMO}(C ; Z)$.

The proof is identical to the one of Theorem 3.3 .

Open Problem 6. Find the analog of Theorem 3.1 when $\mathbb{S}^{1}$ and $\mathbb{R}$ are replaced by $N$ and $Z$.

The case $s p \geq n$ can be treated as in the proof of Theorem 3.1. Probably, the same holds for $s p<1$. It is unclear how to deal with the remaining case $1 \leq s p<n$. Some partial results on this question were obtained by Chiron in an unpublished work.

\section{Approximation}

In this section, $M$ and $N$ are compact Riemannian manifolds without boundary, respectively $n$ and $k$-dimensional. In order to simplify the presentation, we will sometimes replace $M$ by $\mathbb{R}^{n}$ or a domain in $\mathbb{R}^{n}$. Set $X=W^{s, p}(M ; N)$.

A natural question is whether $Y=C^{\infty}(M ; N)$ is dense in $X$. VMO arguments imply that the answer is yes if $s p \geq n$. Therefore, in the remaining part of this section we assume that $s p<n$. Under this assumption, in general the answer is no [SU]: let $u: B^{2} \rightarrow \mathbb{S}^{1}, u(z)=z /|z|$. Then $u \in W^{1,1}$, but $u$ cannot be approximated in $W^{1,1}$ by smooth $\mathbb{S}^{1}$-valued maps. Argue by contradiction: let $\left\{u_{k}\right\} \subset C^{\infty}\left(\overline{B^{2}} ; \mathbb{S}^{1}\right)$ converge to $u$ in $W^{1,1}$. Up to a subsequence, $\left\{u_{k}\right\}$ will converge to $u$ in $W^{1,1}$ (thus uniformly) on almost each circle $C(0, r)$ with $0<r<1$. For any such $r$, the degrees of $u_{k}$ on $C(0, r)$ (which are zero) tend to the degree of $u$ on $C(0, r)$ (which is one)!

Far from closing the subject, this example opens

Direction 1. Characterize $s, p, M$ and $N$ for which $Y$ is dense.

Direction 2. Find "almost smooth" maps that are dense in $X$.

Direction 3. Characterize the closure of $Y$ in $X$.

\subsection{Approximation methods}

We explore here Direction 2. In many situations, it is known that maps in $X$ can be approximated with maps that are smooth except a "small" set $A$ : A could be a finite number of points, or curves,..., up to a finite union of smooth $(n-1)$-dimensional submanifolds of $M$. We describe below the main approximation methods and the results that can be obtained via them. 


\subsubsection{Projection method}

It was first devised, in a different context, by Federer and Fleming [FF]. For approximation purposes, it was first used by Hardt, Kinderlehrer and Lin [HKL1]. This method requires $N=\mathbb{S}^{k}$ (or homeomorphic to $\left.\mathbb{S}^{k}\right)$. It works as follows: let $u \in X$ and $u_{\varepsilon}=u * \rho_{\varepsilon}$. The idea is to project $u_{\varepsilon}$ (which is $\mathbb{R}^{k+1}$-valued) onto $\mathbb{S}^{k}$. For this purpose, let $a \in \mathbb{R}^{k+1}$ with $|a|<1 / 2$ and let $\pi_{a}$ be the projection of $\mathbb{R}^{k} \backslash\{a\}$ onto $\mathbb{S}^{k}$ : thus $\pi_{a}(x)=a+\lambda(x-a)$, where $\lambda>0$ satisfies $|a+\lambda(x-a)|=1$. Let $u_{\varepsilon, a}=\pi_{a} \circ u_{\varepsilon}$. (Note that, if $\left|u_{\varepsilon}\right|$ happens to be close to 1 , then $u_{\varepsilon, a}$ is close to $u_{\varepsilon}$ and thus to $u$.) Almost each $a$ is a regular value for $u_{\varepsilon}$. For such $a, u_{\varepsilon, a}$ is smooth outside the level set $u_{\varepsilon}^{-1}(a)$, which is $(n-k-1)$-dimensional if $n>k$, empty if $n \leq k$. Hopefully, if we choose well $a=a(\varepsilon)$, then $v_{\varepsilon}=u_{\varepsilon, a(\varepsilon)}$ will converge to $u$ in $W^{s, p}$, providing thus an approximation of $u$ with maps that are smooth outside $(n-k-1)_{+}$-dimensional submanifolds of $M$.

This is true if $n \leq k[\mathrm{E}]$; the proof is the same as the one of Theorem 2.1. When $n>k$, partial answers are known when $k \leq s p<k+1$.

Theorem 4.1. Assume that $n>k$ and $k \leq s p<k+1$, and set $l=n-k-1$. Then the class

$$
\mathcal{R}=\left\{u \in C^{\infty}\left(M \backslash A ; \mathbb{S}^{k}\right) ; A=\text {-dimensional submanifold of } M\right\}
$$

is dense in $X$ when:

a) $[B Z] s$ is an integer;

b) $[B B M 2] s<1$;

c) $[\mathrm{Bo}] 1<s<2$.

Actually, [BZ] deals only with $s=1$, but their method works for integer $s$. On the other hand, [BBM2] treats only the case $s=1 / 2, p=2, k=1$ but the proof applies to each $s<1$ and $k$. It is very plausible that Bousquet's method [Bo] gives that the above theorem holds for each $s$. The most delicate case is $s p=k$; for non integer $s$, the first result in this direction was obtained by Rivière [R].

Proof. We only treat the case $s=1$ (and thus $k \leq p<k+1$ ); the case where $s$ is not an integer is more tricky. It is easy to see that $\left|D \pi_{a}(x)\right| \leq C /|x-a|$ uniformly in $x$ and $a$; therefore,

$$
\left|D u_{\varepsilon, a}\right| \leq C\left|D u_{\varepsilon}\right| /\left|u_{\varepsilon}-a\right|
$$

Since $u_{\varepsilon}$ is smooth, this implies that, for $a$ regular value of $u_{\varepsilon}$, we have $\left|D u_{\varepsilon, a}(x)\right| \leq C_{\varepsilon, a} / \operatorname{dist}(x, A)$, where $A=A_{\varepsilon, a}=u_{\varepsilon}^{-1}(a)$. Since $A$ is smooth and $l$-dimensional, we obtain $u_{\varepsilon, a} \in W^{1, p}$ (and even better). Let $B=\bar{B}(0,3 / 4) \subset \mathbb{R}^{k+1}$ and let $K=K_{\varepsilon, a}=u_{\varepsilon, a}^{-1}(B)$. Outside $B$, we are far away form $a$, and thus $\pi_{a}$ is Lipschitz uniformly in $a$. Since $u=\pi_{a} \circ u$, we have $u_{\varepsilon, a}-u=\pi_{a} \circ u_{\varepsilon}-\pi_{a} \circ u$; from this, we find easily that $\left\|u_{\varepsilon, a}-u\right\|_{W^{1, p}(M \backslash K)} \rightarrow 0$ with $\varepsilon$, and this holds uniformly in $a$. The choice of $a$ comes into the picture when we want to prove convergence in $K$. If we integrate (4.1) over $K_{\varepsilon, a}$, then in $a$, we find

$$
\iint_{K}\left|D u_{\varepsilon, a}\right|^{p} d x d a \leq C \iint_{K}\left|D u_{\varepsilon}\right|^{p} /\left|u_{\varepsilon}-a\right|^{p} d x d a \leq C \iint_{K}\left|D u_{\varepsilon}\right|^{p} d x d a
$$

the last inequality holds since $p<k+1$, and thus $\int_{B^{k+1}}|a-x|^{-p} d a \leq C<\infty$. Using the fact that $\left|K_{\varepsilon, a}\right| \rightarrow 0$ with $\varepsilon$, it follows that the last term in (4.2) tends to 0 . Thus, we may pick regular values $a$ such that $\left\|u_{\varepsilon, a}-u\right\|_{W^{1, p}(K)} \rightarrow 0$.

It is natural to try to remove the assumption $s p<k+1$ in Theorem 4.1. However, the natural conjecture is that the singular set should be not $(n-k-1)$, but rather $(n-[s p]-1)$-dimensional. 
Open Problem 7. Assume that $n>k+1$ and $k+1 \leq s p<n$, and let $l=n-[s p]-1$. Is it true that the class

$$
\mathcal{R}=\left\{u \in C^{\infty}\left(M \backslash A ; \mathbb{S}^{k}\right) ; A=\text { finite union of l-dimensional submanifolds of } M\right\}
$$

is dense in $X$ ?

Same question when $\mathbb{S}^{k}$ is replaced by an arbitrary $N$.

In view of the projection method, it is a bit surprising that $k$ has to be replaced by $[s p]$. We will see why in Sections 4.1.2 and 4.1.3.

The answer to Open Problem 7 is yes when $k=1$ [BBM4]. Actually, in this case $C^{\infty}\left(M ; \mathbb{S}^{1}\right)$ is dense in $X$.

\subsubsection{Good and bad cubes method}

It is due to Bethuel [Be2] and works for $s=1$ (and thus $p<n$ ). For simplicity, we let $M=\mathbb{R}^{n}$.

We treat here the case $n-1<p<n$. We will show that maps in $W^{1, p}\left(\mathbb{R}^{n} ; N\right)$ can be approximated with maps smooth except at a finite number of points.

Fix $\varepsilon>0$ and $y \in(0, \varepsilon)^{n}$. The cubes $C=C_{m, \varepsilon, y}=y+\varepsilon m+(0, \varepsilon)^{n}$, with $m \in \mathbb{Z}^{n}$, cover $\mathbb{R}^{n}$; let $\mathcal{F}=\mathcal{F}_{\varepsilon, y}$ be the collection of these cubes. The $(n-1)$-dimensional skeleton $\mathcal{C}_{n-1}$ of $\mathcal{F}$ is the union of the faces of the cubes. The $(n-2)$-skeleton $\mathcal{C}_{n-2}$ is the union of the boundaries of these faces, and so on. The 0 -skeleton $\mathcal{C}_{0}$ is formed simply by the vertexes of the cubes.

By Fubini, one may find $y=y(\varepsilon)$ such that $|u|_{W^{1, p}\left(\mathcal{C}_{n-1}\right)} \leq C / \varepsilon$. For such $y, u$ is in $W^{1, p}$ (thus Hölder continuous) on $\mathcal{C}_{n-1}$. The idea is to approximate $u$ on each cube $C$ without modifying $u$ on $\partial C$, and to glue these approximations. The way the approximation is performed on $C$ depends on how much $u$ oscillates on $C$.

With $\delta>0$ small, a cube $C$ is "bad" (=u oscillates a lot on $C)$ if $|u|_{W^{1, p}(C)} \geq \delta \varepsilon^{n-p+1}$ or $|u|_{W^{1, p}(\partial C)} \geq$ $\delta \varepsilon^{n-p-1}$; the choice of $y$ implies that the union $A=A_{\varepsilon}$ of bad cubes is small (its measure tends to 0 with $\varepsilon)$. The remaining cubes are "good".

On a bad cube, the main care is to construct a map with few singularities and not too large norm; since there are few bad cubes, this will suffice. E. g., one may consider a solution $v$ of min $\left\{\int_{C}|D w|^{p} ; w\right.$ : $C \rightarrow N, w=u$ on $\partial C\}$; thus $v$ has less energy than $u$. By a deep regularity result [HL], $v$ is continuous in $\bar{C}$ except finitely many points in $C$. It is easy to see that $\int_{A}|D u-D v|^{p} \rightarrow 0$.

If $C$ is good and $\delta$ sufficiently small, then the image of $\partial C$ is contained in some small ball $B$, by the Sobolev embeddings; the center of $B$ will depend on $C$, but not its radius $r$. If $\tilde{B}$ is the ball concentric to $B$ and twice larger, it is possible to project $N$ on $\tilde{B} \cap N$ through a map $\Phi$, Lipschitz uniformly in $B$ (flatten locally $N$, next take the nearest point projection; this is where smallness of balls is needed). On $C$, we approximate $u$ with $v_{1}=\Phi \circ u$, which agrees with $u$ on $\partial C$. The map $v_{1}$ is $\tilde{B}$-valued. Morally, this means that $v_{1}$ is $\mathbb{R}^{k}$-valued; a standard technique allows then to approximate $v_{1}$ with a continuous map $v$ agreeing with $u$ on $\partial C$. (A similar argument appeared in the proof of Theorem 2.1.) One has to check next that $v_{1}$ is close to $u$. These two maps differ only on the set $D$ where $\Phi(u(x)) \neq u(x)$. If $x \in D$ and if $C$ is the good cube containing $x$, then

$$
|u|_{W^{1, p}(E)} \geq \delta \varepsilon^{n-p-1}, \quad \text { where } E=\left\{z \in C ; z_{n}=x_{n}\right\} .
$$

Argue by contradiction: if not, $u(E)$ is contained in a ball of size $r$ (by Sobolev embeddings), and this ball intersects $B$, since $u(\partial E) \subset B$. Thus $u(E) \subset \tilde{B}$ ! The choice of $y$ and (4.3) imply that $|D| \rightarrow 0$, 
so that $\left|u-v_{1}\right|_{W^{1, p}(D)} \leq C|u|_{W^{1, p}(D)} \rightarrow 0$.

To summarize, $u$ has been approximated with maps $v$ that are continuous except finitely many points. By further mollifying $v$, one may find an approximation with maps which are $C^{\infty}$ except a finite set. The remaining case $1 \leq p \leq n-1$ requires additional ingredients. For a relatively short proof in this case, see Hang and Lin [HaL2], Section 6. The result obtained through this method is

Theorem 4.2. Let $1 \leq p<n=\operatorname{dim} M$ and $l=n-[p]-1$. Then

$$
\mathcal{R}=\left\{u \in C^{\infty}(M \backslash A ; N) ; A=\text { finite union of l-dimensional submanifolds of } M\right\}
$$

is dense in $W^{1, p}(M ; N)$.

Gluing is a key ingredient in the proof. In $W^{1, p}$, this works since two $W^{1, p}$ maps defined on neighbor cubes which have the same trace on their common face are in $W^{1, p}$ of the union of cubes. This leaves the hope of adapting this argument in $W^{s, p}$ for $s<1+1 / p$; when $s>1+1 / p$, higher order traces appear, and the method needs to be supplemented with entirely new ideas.

\subsubsection{Homogeneous extensions method}

This works only for $s<1$ and is taken from [BBM4]. We explain it when $M=\mathbb{R}^{n}$ and $s p$ is not an integer. Let $m=[s p]$. For a. e. $y$, the restriction of $u$ to the $m$-skeleton $\mathcal{C}_{m}$ of $\mathcal{F}_{\varepsilon, y}$ is in $W^{s, p}$, thus continuous. For any such $y$, we define $u_{y}=u_{y, \varepsilon}$ as follows: on $\mathcal{C}_{m}, u_{y}=u$. Assuming $u_{y}$ defined on $\mathcal{C}_{j}$, $j<n$, we proceed to define $u_{y}$ on an arbitrary face $F$ of $\mathcal{C}_{j+1}$. If $z$ is the center of $F$ and $t \in \partial F$, we take $u_{y} \equiv u_{y}(t)$ on the whole segment from $z$ to $t$. (This is the "homogeneous extension" technique.) We extend in the same way $u_{y}$ from $\mathcal{C}_{n-1}$ to $\mathbb{R}^{n}$, and we end with a map defined in $\mathbb{R}^{n}$. If, for example, $m=n-1$, then $u_{y}$ is continuous except at the centers of the cubes $C$. In general, $u_{y}$ is continuous except a countable union of $(n-m-1)$-planes. Next, the key ingredient is the estimate

$$
f_{(0, \varepsilon)^{n}}\left|u_{y, \varepsilon}-u\right|_{W^{s, p}}^{p} d y \rightarrow 0 \quad \text { as } \varepsilon \rightarrow 0,
$$

valid when $s p<n$ and $s<1$. It implies that we may find $y=y(\varepsilon)$ such that $u_{y(\varepsilon), \varepsilon} \rightarrow u$ in $W^{s, p}$. Further mollification allows to replace the $u_{y}$ 's by maps that are $C^{\infty}$ outside a countable union of $(n-m-1)$ planes, and thus prove the following

Theorem 4.3. Let $s p<n=\operatorname{dim} M, s<1$ and $l=n-[s p]-1$. Then

$$
\mathcal{R}=\left\{u \in C^{\infty}(M \backslash A ; N) ; A=\text { finite union of l-dimensional submanifolds of } M\right\}
$$

is dense in $W^{s, p}(M ; N)$.

Since gluing is part of the method, one may hope to use it for $s<1+1 / p$. This will not work, even for $s=1$ : for $u \in C_{0}^{\infty} \backslash\{0\}$, we have $\left|D\left(u_{y}-u\right)\right|_{L^{p}} \geq C>0$.

\subsection{Density of smooth maps}

We step forward Direction 1: density of $Y=C^{\infty}(M ; N)$ in $X=W^{s, p}(M ; N)$. The main known result concerns $W^{1, p}$. It was obtained in [Be2], but both the statement and the proof were incomplete. The corrected result is due to Hang and Lin [HaL2]. It is simple to state only when $n-1 \leq p<n$.

Theorem 4.4. Let $n-1 \leq p<n=\operatorname{dim} M$. Then $Y=C^{\infty}(M ; N)$ is dense in $X=W^{1, p}(M ; N)$ if and only if the homotopy group $\pi_{n-1}(N)$ is trivial. 
Proof. Assume that $\pi_{n-1}(N) \neq\{0\}$; we will construct a map $u$ which cannot be approximated with smooth maps. There is a map $v \in C^{\infty}\left(B^{n-1} ; N\right)$, identically equal to a constant $C$ near $\mathbb{S}^{n-2}$, and such that $v$ is not homotopic to a constant. After locally flattening $M$, we may assume that $M$ contains $B^{n}$. Let $D$ (respectively $E$ ) be the cone with vertex the North Pole (respectively South Pole) of $\mathbb{S}^{n-1}$ and base $B^{n-1}$, and set $F=D \cup E$. On each horizontal slice $S$ of $F$, we may transport $v$ by translation and dilation. If $u$ is the map obtained in this way, then, for $-1<t<1$, the restriction of $u$ to $F \cap\left\{x_{n}=t\right\}$ is smooth and not homotopic to a constant. We extend $u$ to $M$ with the value $C$ outside $F$; then $u \in W^{1, p}$. Assume now by contradiction that $Y$ is dense in $X$. Possibly after passing to a subsequence, we find $\left\{u_{k}\right\} \subset C^{\infty}(M ; N)$ such that, on a. e. $S, u_{k} \rightarrow u$ in $W^{1, p}$, thus in VMO. The homotopy class being stable with respect to VMO convergence (cf Section 2.7), we find that, for large $k, u_{k \mid S}$ is not homotopic to a constant, which is absurd.

Conversely, assume that $\pi_{n-1}(N)=\{0\}$. It suffices to prove that maps in $\mathcal{R}$ can be approximated by smooth maps. We explain the method when $u$ has only one singularity; in the general case, we apply this procedure near each singular point. We may assume that $M$ contains $B^{n}$, and that the singular point is the origin. For $0<r<1$, we extend $u$ from $S(0, r)$ to $B(0, r)$ by homogeneous extension (thus the extension $v_{r}$ is constant on rays). Let $u_{r}$ be the map that equals $u$ outside $B(0, r)$ and $v_{r}$ inside $B(0, r)$. By a Fubini type argument, there is a sequence $r_{n} \rightarrow 0$ such that $u_{r_{n}} \rightarrow u$ in $W^{1, p}$. It suffices thus to consider maps $u$ which are smooth in $M \backslash B(0, r)$ and homogeneous in $B(0, r)$; assume, e. g., that $r=1$. The map $v=u_{\mid \mathbb{S}^{n-1}}$ is smooth and, as a continuous map, homotopic to a constant $C$. By regularization, there is a homotopy $H \in C^{\infty}\left([0,1] \times \mathbb{S}^{n-1} ; N\right)$ such that $H(\cdot, 1)=v$

and $H(\cdot, t)=C$ for $t \leq 1 / 2$. For $0<\varepsilon<1$, we define $u_{\varepsilon}(x)=\left\{\begin{array}{ll}u(x) & \text { outside } B(0, \varepsilon) \\ H(t|x| / \varepsilon, x /|x|) & \text { in } B(0, \varepsilon)\end{array}\right.$. It is easy to see that $u_{\varepsilon} \rightarrow u$ in $W^{1, p}$. Since the $u_{\varepsilon}$ 's are continuous, they can be approximated with smooth maps.

When $1 \leq p<n-1$, the condition $\pi_{[p]}(N)=\{0\}$ is necessary, but not sufficient for density; we send to [HaL2], Section 6 for details.

Open Problem 8. Find, for arbitrary $s, p M$ and $N$ such that $s p<n=\operatorname{dim} M$, a necessary and sufficient condition for the density of $Y$ in $X$.

The answer is known when $s<1$ [BBM4], and the density condition depends on the value of $s p$. For example, the analog of Theorem 4.4 is

Theorem 4.5. Assume that $n-1 \leq s p<n$. Then $Y$ is dense in $X$ if and only if $\pi_{n-1}(N)=0$.

The answer is also known when $N=\mathbb{S}^{1}[\mathrm{BBM} 4]$ :

Theorem 4.6. Assume that $N=\mathbb{S}^{1}$. Then $Y$ is dense in $X$ except when $n \geq 2$ and $1 \leq s p<2$.

We emphasize the fact that even for the space $W^{2, p}$ the answer is not known. It is quite likely that understanding this case will unblock the general situation.

\subsection{The singular set of a map}

Assume that $Y$ is not dense in $X$, but that we are able to approximate a map $u \in X$ with maps $u_{k}$ in the class $\mathcal{R}$. Question: can one "pass to the limits" the singular sets of the $u_{k}$ 's? If so, one has a natural notion of singular set of $u$, and can even dream of proving that $u$ is in the closure of $Y$ if and only its singular set is empty. (Thus this question is related to Direction 3.) Most of the work in his direction has been done when $N=\mathbb{S}^{k}$. Except at the very end of this this section, we let $N=\mathbb{S}^{k}$. To 
start with, we take $n=k+1$ and $M=\mathbb{S}^{k+1}$; however, $M$ could be any $(k+1)$-dimensional manifold. The maps we consider are $W^{1, p}$, with $k \leq p<k+1$. (When $p$ is not in this range, $Y$ is dense in $X$.) Actually, it suffices to know how to pass to the limits the singular set when $u \in W^{1, k}$; we take thus $p=k$. In this case, $\mathcal{R}$ consists of maps $u$ smooth outside some finite set $A=A(u)$. To each $a \in A$, we may associate a degree, defined as the degree of $u$ on a small geodesic sphere around $a$ on $\mathbb{S}^{n}$, positively oriented with respect to the outward normal at $a$; this integer is independent of the small sphere and will be denoted $\operatorname{deg}(u, a)$. Brezis, Coron and Lieb [BCL] discovered the fact that the singular set $A$ can be obtained from $u$ via an analytic formula. More specifically, if $T=T_{u}=\sum_{a \in A} \operatorname{deg}$ $(u, a) \delta_{a}$ and $c_{k}=1 /\left|\mathbb{S}^{k}\right|$, then the action of the distribution $T$ is given by

$$
T(\zeta)=-c_{k} \sum \int_{\mathbb{S}^{k+1}} \partial_{j} \zeta \operatorname{det}\left(\partial_{1} u, \ldots, \partial_{j-1} u, u, \partial_{j+1} u, \ldots, \partial_{k+1} u\right) .
$$

Here, the derivatives are computed in an orthogonal positively oriented frame. Note that the righthand side of (4.4) makes sense for $u \in W^{1, k}$. If we endow $W^{1, \infty}\left(\mathbb{S}^{k+1} ; \mathbb{R}\right)$ with the semi-norm $\zeta \mapsto$ $\|D \zeta\|_{L^{\infty}}$, then $T$ given by (4.4) lies in $\left(W^{1, \infty}\right)^{*}$, and depends continuously on $u$. Since each $u$ may be approximated by maps in $\mathcal{R}$, one may intuitively think of $T$ as an infinite sum of Dirac masses. This is indeed correct.

Proposition 4.1. For $u \in W^{1, p}\left(\mathbb{S}^{k+1} ; \mathbb{S}^{k}\right)$, one may write $T_{u}=\sum\left(\delta_{P_{i}}-\delta_{N_{i}}\right)$, for two sequences $\left\{P_{i}\right\},\left\{N_{i}\right\} \subset \mathbb{S}^{k+1}$ such that $\left|P_{i}-N_{i}\right|<\infty$.

Proof. Assume $u \in \mathcal{R}$. Then $\sum_{a \in A} \operatorname{deg}(u, a)=0$. This may be seen either from topological considerations, or by noting that $T(1)=0$. Thus the points in $A$ counted with the multiplicity of their degree (a point with degree 2 appears twice as a "positive point", a point with degree -1 appears once as a "negative point", a point of degree 0 does not appear at all) form a list $P_{1}, \ldots P_{m}, N_{1}, \ldots, N_{m}$ of positive and negative points, the positive points being as many as the negative ones. With the points in $A$ listed in this way, we have $T(\zeta)=\sum_{i=1}^{m}\left(\zeta\left(P_{i}\right)-\zeta\left(N_{i}\right)\right)$. The key ingredient is the following sup-inf inequality devised in $[\mathrm{BCL}]$ :

$$
\sup \left\{\sum_{i=1}^{m}\left(\zeta\left(P_{i}\right)-\zeta\left(N_{i}\right)\right) ;|D \zeta| \leq 1\right\}=\inf _{\sigma \in S_{m}} \sum d\left(P_{i}, N_{\sigma(i)}\right)
$$

here, $d$ is the geodesic distance on $\mathbb{S}^{k+1}$ and $S_{m}$ is the $m$ th symmetric group.

Formula (4.4) gives $\left|T_{u}(\zeta)\right| \leq C\|D u\|_{L^{k}}^{k}\|D \zeta\|_{L^{\infty}}$. In view of (4.5), $T$ may be written as $T(\zeta)=$ $\sum\left(\zeta\left(P_{i}\right)-\zeta\left(N_{i}\right)\right)$ with $\sum d\left(P_{i}, N_{i}\right) \leq C\|D u\|_{L^{k}}^{k}$. A Cauchy sequences argument, combined with the fact that the geodesic distance is equivalent to the Euclidean one, allows to conclude.

It is tempting to consider the set $\left\{P_{i}\right\} \cup\left\{N_{i}\right\}$ as the singular set of $u$. This is not realistic, since there is a high degree of non uniqueness in the choice of these points; see Ponce [P1] for a thorough discussion on the infinite sums of Dirac masses. In a somehow non intuitive way, one has to identify the singular set of $u$ with the distribution $T_{u}$; when $u \in \mathcal{R}, T_{u}$ can further be identified with a set of points.

Proposition 4.1 has a converse [ABO]: given sequences $\left\{P_{i}\right\},\left\{N_{i}\right\} \subset \mathbb{S}^{k+1}$ such that $\left|P_{i}-N_{i}\right|<\infty$, there is a map $u \in W^{1, k}\left(\mathbb{S}^{k+1} ; \mathbb{S}^{k}\right)$ such that $T_{u}=\sum\left(\delta_{P_{i}}-\delta_{N_{i}}\right)$. The map $u$ is explicitly constructed using the "dipole construction" in [BCL]. All the above results can be summed in the following 
Proposition 4.2. The map $u \mapsto T_{u}$ is continuous from $W^{1, k}\left(\mathbb{S}^{k+1} ; \mathbb{S}^{k}\right)$ into $\left(W^{1, \infty}\right)^{*}$, and its range is $\left\{\sum_{\text {finite }}\left(\delta_{P_{i}}-\delta_{N_{i}}\right)\right\}^{\left(W^{1, \infty}\right)^{*}}$.

It turns out that one may define $T_{u}$ for $u \in W^{s, p}$ when $k \leq s p<k+1$ [BBM3]. By Sobolev and Gagliardo-Nirenberg, it suffices to consider the case $s p=k, s<1$.

Theorem 4.7. Assume that $s p=k$ and $s<1$. Then the map $u \mapsto T_{u}$, initially defined for $u \in \mathcal{R}$, extends by density as a continuous map from $W^{s, p}\left(\mathbb{S}^{k+1} ; \mathbb{S}^{k}\right)$ into $\left(W^{1, \infty}\right)^{*}$, and its range

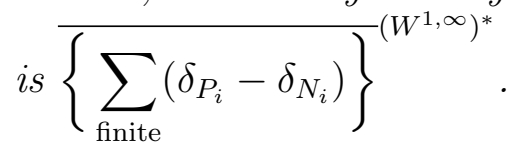

Proof. Formula (4.4) does not make sense for $u \in W^{s, p}$. The idea is to find another formula for $T_{u}$; this is very much in the spirit of Section 2.2. We take $v$ as there, i. e., $v=\left\{\begin{array}{l}\tilde{u} /|\tilde{u}|, \text { if }|\tilde{u}| \leq 1 / 2 \\ 2 \tilde{u}, \text { if }|\tilde{u}| \leq 1 / 2\end{array}\right.$, with $\tilde{u}$ the harmonic extension of $u$. Let also $\xi$ be any smooth extension of $\zeta$ to $B^{k+2}$. For $u$ in $W^{1-1 /(k+1), k+1} \cap W^{1, k}, u$ smooth outside a finite set (call such a $u$ a good map), we have, with $d_{k}=(k+1) c_{k}$,

$$
T_{u}(\zeta)=d_{k} \sum(-1)^{k+j} \operatorname{det}\left(\partial_{1} v, \ldots, \partial_{j-1} v, \partial_{j+1} v, \ldots, \partial_{k+2} v\right) \partial_{j} \xi
$$

this can be easily checked for smooth $u$, next by approximation. Since good maps are dense in $W^{s, p}\left(\mathbb{S}^{k+1} ; \mathbb{S}^{k}\right)$, it suffices to prove that the right-hand side of (4.6) depends continuously on $u$ and $\xi$; this is done as in the proof of Theorem 2.3. Finally, the range of $u \mapsto T_{u}$ is determined by adapting the dipole construction.

While the above result allows to define $T_{u}$ in $W^{s, p}$ if $k<s p<k+1$, it says nothing about the set of all the $T_{u}$ 's.

Open Problem 9. Assume that $k<s p<k+1$. Characterize the distributions $T$ which are of the form $T=T_{u}$ for some $u \in W^{s, p}\left(\mathbb{S}^{k+1} ; \mathbb{S}^{k}\right)$.

The answer is not known even when $s=1$. Partial results were obtained, for $k=1$, by Bousquet

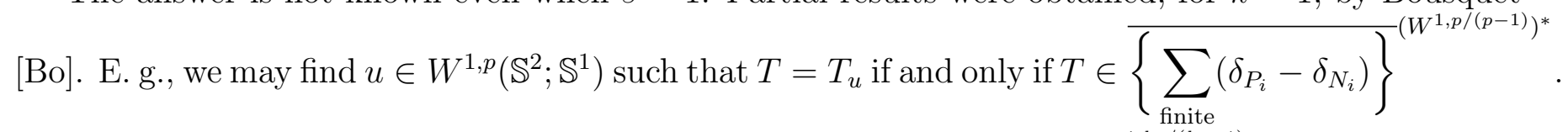
Presumably, in $W^{1, p}\left(\mathbb{S}^{k+1} ; \mathbb{S}^{k}\right)$ the range of $u \mapsto T_{u}$ is $\left\{\sum_{\text {finite }}\left(\delta_{P_{i}}-\delta_{N_{i}}\right)\right\}^{\left(W^{1, k p /(k p-1)}\right)^{*}}$.

The following result, due to Bethuel [Be1], suggests that $T_{u}$ really describes the singular set of $u$.

Theorem 4.8. Let $u \in X=W^{1, k}\left(\mathbb{S}^{k+1} ; \mathbb{S}^{k}\right)$. Then $u \in Y$ if and only if $T_{u}=0$.

Proof. When $u \in C^{\infty}$, we have $T_{u}=0$; by continuity, $T_{u}=0$ if $u \in Y$. The key ingredient in the proof of the converse is the following result, whose proof relies on an explicit construction similar to the dipole one.

Lemma 4.1. If $A$ is the singular set of $u \in \mathcal{R}$, then there is a map $v \in W^{1, k}\left(\mathbb{S}^{k+1} ; \mathbb{S}^{k}\right)$, locally Lipschitz outside $A$, such that $\operatorname{deg}(v, a)=0, a \in A$, and $\|D(u-v)\|_{L^{k}}^{k} \leq C\left\|T_{u}\right\|_{\left(W^{1, \infty}\right)^{*}}$. 
Restricted to a small geodesic sphere $S$ around some $a \in A$, the above $v$ has degree 0 ; thus $v_{\mid S}$ is homotopic to a constant. The proof of Theorem 4.4 shows that $v \in Y$. Lemma 4.1 implies that $\operatorname{dist}(u, Y) \leq C\left\|T_{u}\right\|_{\left(W^{1, \infty}\right)^{*}}^{1 / k}$ (here, the distance is computed with respect to the $W^{1, k}$-semi-norm). By continuity, this inequality holds for each $u \in W^{1, k}$. This completes the proof.

The proof of Lemma 4.1 works only for $p=k$. It can be adapted in $W^{s, p}$, but only if $s p=k$ (see, e. g., [BBM2]). This leaves us with the following

Open Problem 10. Assume that $k \leq s p<k+1$ and let $u \in W^{s, p}\left(\mathbb{S}^{k+1} ; \mathbb{S}^{k}\right)$. Is it true that $u \in Y$ if and only if $T_{u}=0$ ?

The answer is yes when $s \leq 1$ Ponce [P2] or when $k=1$ [Bo].

We next consider maps in $W^{1, k}\left(\mathbb{S}^{n} ; \mathbb{S}^{k}\right)$, with $n>k+1$. In this case, the singular set of a map $u \in \mathcal{R}$ is an $(n-k-1)$-dimensional manifold $A$. We may still associate to $u$ an object $T_{u}$, but this time it acts on $(n-k-1)$-forms (=sections of $\Lambda^{n-k-1}\left(T^{*}\left(\mathbb{S}^{n}\right)\right)$, not on functions. For simplicity, we explain how this is done when $n=k+2$. (In higher dimensions, the ideas are the same [JS], [HaL1], [ABO].) The singular set $A$ of $u$ is a finite union of compact simple curves. To start with, assume that $A$ consists of only one curve, say $\Gamma$. We choose an orientation on $\Gamma$ and let $\tau$ be the tangent unit vector positively oriented on $\Gamma$. We may define the degree of $u$ around $\Gamma$ as follows: we take an $(n-1)$-submanifold $P$ of $\mathbb{S}^{n}$, transversal to $\Gamma$ at some point $x$, and oriented positively with respect to $\Gamma$ (i. e., the orientation on $T_{x}(\Gamma) \times T_{x}(P)$ is the positive one on $\mathbb{S}^{n}$ ). This orientation induces a positive orientation on small geodesic spheres $S$ on $P$ around $x$. We define $\operatorname{deg}(u, \Gamma)=\operatorname{deg} u_{\mid S}$; this integer does not depend on $x$ or $S$. Then the object associated to $u$ is $T=T_{u}=\operatorname{deg}(u, \Gamma) \tau \delta_{\Gamma}$, with $\delta_{\Gamma}$ the Dirac mass on $\Gamma$ (=the 1-dimensional Hausdorff measure restricted to $\Gamma$ ). When $A=\cup \Gamma_{i}$, we let $T=\sum \operatorname{deg}\left(u, \Gamma_{i}\right) \tau \delta_{\Gamma_{i}}$. This object acts on 1-forms, i. e. on smooth sections $\omega$ of the cotangent bundle to $\mathbb{S}^{n}$ through the formula $T(\omega)=\sum \operatorname{deg}\left(u, \Gamma_{i}\right) \int_{\Gamma_{i}}<\omega, \tau>d s$. (In case of $p$-dimensional manifolds, $p \geq 2$, one defines similarly $T$ through the formula $T(\omega)=\sum \operatorname{deg}\left(u, \Gamma_{i}\right) \int_{\Gamma_{i}}<\omega, \tau>$; here, $\tau$ is a unit $p$-vector positively oriented, and $\omega$ is a $p$-form.)

If we write, in an orthogonal positively oriented frame, $\omega=\sum \omega_{i} d x_{i}$ and we set $\omega_{i j}=\partial_{i} \omega_{j}-\partial_{j} \omega_{i}$, then the analog of (4.4) is

$$
T(\omega)=-c_{k} \sum_{i<j} \int_{\mathbb{S}^{n}} \omega_{i j} \operatorname{det}\left(\partial_{1} u, . ., \partial_{i-1} u, u, \partial_{i+1} u, . ., \partial_{j-1} u, \partial_{j+1} u, . ., \partial_{n} u\right) .
$$

If $u$ is a good map, then we also have an analog of (4.6): with $v$ as in (4.6), $\Omega$ a smooth extension of $\omega$, and $a_{i}=d_{k}(-1)^{k+i}$,

$$
T(\omega)=\sum_{i<j} a_{i} \int_{B^{n+1}} \Omega_{i j} \operatorname{det}\left(\partial_{1} v, . ., \partial_{i-1} v, \partial_{i+1} v, . ., \partial_{j-1} v, \partial_{j+1} v, . ., \partial_{n+1} v\right) .
$$

Recall that, in case of point singularities, positive and negative points in $A$ are in equal number. This may be translated as $A=\partial C$, where $C$ is a union of curves in $\mathbb{S}^{n}$, each one with starting point a negative point and endpoint a positive point. Here, the boundary has to be understood in the distributions sense: if we orientate each curve from the negative to the positive point, then it defines a current (still denoted $C$ ) as above, and the equality $A=\partial C$ means $T_{u}(\zeta)=C(d \zeta), \forall \zeta$. The counterpart of these properties in the case of curves is that that the $\Gamma_{i}$ 's are closed, which in turn 
implies that we may write $T_{u}=\partial S$, where this time $S$ is the current associated to a finite union of surfaces. The analog of (4.4) is

$$
\sup \left\{T_{u}(\omega) ;|d \omega| \leq 1\right\}=\inf \{|S| ; \partial S=T\}
$$

here, $|S|$ stands for the mass (=surface) of $S$. Once the parallel between the two situations is established, the counterpart of Proposition 4.2 is $[\mathrm{ABO}]$

Theorem 4.9. The map $u \mapsto T_{u}$ is continuous from $W^{1, k}\left(\mathbb{S}^{k+2} ; \mathbb{S}^{k}\right)$ into the dual of Lipschitz 1-forms, and its range is $\{\partial S ; S$ is a rectifiable surface $\}$.

The delicate part of the proof is the construction of $u$ when $S$ is given; this is done by adapting carefully the dipole construction. A similar statement holds in higher dimensions.

A straightforward adaptation of the proof of Theorem 4.7 gives the existence of $T_{u}$ when $u \in W^{s, p}$ and $s p=k$. Thus one may consider Open Problems 9 and 10 when $\mathbb{S}^{k+1}$ is replaced by $\mathbb{S}^{n}$, with $n \geq k+2$. We end this section by considering the case where $\mathbb{S}^{k}$ is replaced by a general $k$-dimensional manifold $N$. If $n=k+1$ and $M$ is $n$-dimensional, then maps $u$ with point singularities are dense in $W^{1, k}(M ; N)$. If $A$ is the singular set of $u$, it is natural to associate to $a \in A$ a homotopy class $[u](a)$, namely the class of $u$ restricted on a small geodesic sphere around $a$. The proof of Theorem 4.4 shows that $u \in Y$ if and only if $[u](a)$ is trivial for each $a$. It is not known whether one can associate to $u$ a distribution that "hears" the singularities of $u$.

Open Problem 11. Is there a way to associate to a map u with point singularities a distribution $T_{u}$ supported in the singular set of $u$, depending continuously on the $W^{1, k}$-norm and such that $T_{u}=0$ if and only if $u \in Y$ ?

\subsection{Relaxed energy}

Though the questions raised in this section make sense for general manifolds $M$ and $N$, we shall consider only $M=\mathbb{S}^{k+1}$ and $N=\mathbb{S}^{k}$; even this special case is not well-understood. If $k \leq s p<k+1$, then smooth maps are not dense in $X=W^{s, p}\left(\mathbb{S}^{k+1} ; \mathbb{S}^{k}\right)$. However, one may hope weak density of smooth maps, i. e., that given $u \in X$, there is a sequence $\left\{u_{k}\right\}$ of smooth maps, bounded in $X$, and such that $u_{k} \rightarrow u$ a. e. There is no weak density if $s p>k$. Indeed, let $u\left(x^{\prime}, x_{k+2}\right)=x^{\prime} /\left|x^{\prime}\right|$, which is singular at the poles of $\mathbb{S}^{k+1}$. Argue by contradiction and assume that there is a sequence $\left\{u_{k}\right\}$ as above. Then, up to some subsequence, on a. e. geodesic sphere $S$ around the North Pole of $\mathbb{S}^{k+1}$, we have $u_{k} \rightarrow u$ in $W^{s, k / s}(S)$, and thus in VMO. This leads to a contradiction, since the degree of $u$ on $S$ is 1 , while the one of the $u_{k}$ 's is 0 . In view of this example, from now on $s p=k$. The relaxed energy introduced by Bethuel, Brezis and Coron $[\mathrm{BBC}]$ is "the least energy required to approximate $u$ ":

$$
E_{\text {rel }}(u)=\inf \left\{\liminf \left|u_{k}\right|_{W^{s, p}}^{p} ;\left\{u_{k}\right\} \subset C^{\infty}\left(\mathbb{S}^{k+1} ; \mathbb{S}^{k}\right), u_{k} \rightarrow u \text { a.e. }\right\} .
$$

Clearly, $E_{\text {rel }}(u) \geq|u|_{W^{s, p}}^{p}$. If $\mathcal{R}$ is dense, then the relaxed energy is always finite; this relies on a dipole construction. It is very likely that $\mathcal{R}$ is always dense (cf the discussion after Theorem 4.1). The exact formula of the relaxed energy is known only when $s=1, p=k$. This formula is related to the singular set of $u$ and establishes a bridge between Directions 2 and 3. If $u \in \mathcal{R}$, let $L(u)$ be the right-hand side of (4.5). In a suggestive way, $L(u)$ is called the minimal connection between the negative points $N_{i}$ and the positive points $P_{i}[\mathrm{BCL}]$. For a general $u, L(u)$ is defined as $\left\|T_{u}\right\|_{\left(W^{1, \infty}\right)^{*}}$.

Theorem 4.10. In $W^{1, k}\left(\mathbb{S}^{k+1} ; \mathbb{S}^{k}\right)$, we have

$$
E_{\mathrm{rel}}(u)=\|D u\|_{L^{p}}^{p}+\left|\mathbb{S}^{k}\right| k^{k / 2} L(u) .
$$


Proof. For $k \geq 2$, this result is from [BBC]. We take $k=2$; when $k>2$, the argument is similar. Inequality $\leq$ in (4.11) is established, for $u \in \mathcal{R}$, via the explicit construction of a sequence $\left\{u_{k}\right\}$ such that $u_{k} \rightarrow u$ a. e. and $\left\|D u_{k}\right\|_{L^{2}}^{2} \rightarrow\|D u\|_{L^{2}}^{2}+8 \pi^{2} L(u)$. By density, such a sequence exists also for a general $u$. For $\geq$, the key argument is that, for fixed $\zeta$ with $|D \zeta| \leq 1$, the map $u \mapsto\|D u\|_{L^{2}}^{2}+8 \pi^{2} T_{u}(\zeta)$ is lower semi-continuous on the convex set of $H^{1}$-maps of modulus $\leq 1$. By taking the supremum over $\zeta$, this implies that $u \mapsto\|D u\|_{L^{2}}^{2}+8 \pi^{2} L(u)$ is lower semi-continuous. Thus, for any sequence $\left\{u_{k}\right\}$ such that $u_{k} \rightarrow u$, we have

$$
\|D u\|_{L^{2}}^{2}+8 \pi^{2} L(u) \leq \liminf \left(\left\|D u_{k}\right\|_{L^{2}}^{2}+8 \pi^{2} L\left(u_{k}\right)\right)=\liminf \left\|D u_{k}\right\|_{L^{2}}^{2} .
$$

This argument does not apply when $k=1$; in this case, this result was proved using a different method in [GMS2]; for an elementary proof, we refer to [BMP].

Nothing is known when $s \neq 1$. We end with the following challenging

Open Problem 12. Assume that $0<s<1$ and $s p=k$. Prove that, for $u \in W^{s, p}\left(\mathbb{S}^{k+1} ; \mathbb{S}^{k}\right)$, we have $E_{\mathrm{rel}}(u)=|u|_{W^{s, p}}^{p}+C L(u)$, where $C$ depends only on $s$ and $k$.

The only hint towards this question is the fact that, when $k=1$, we have $E_{\text {rel }}(u)-|u|_{W^{s, p}}^{p} \sim L(u)$ [BBM2].

\section{References}

[ABO] G. Alberti, S. Baldo, G. Orlandi, Functions with prescribed singularities, J. Eur. Math. Soc. 5 (2003), 275-311.

[Be1] F. Bethuel, A characterization of maps in $H^{1}\left(B^{3}, \mathbb{S}^{2}\right)$ which can be approximated by smooth maps, Ann. Inst. H. Poincaré Anal. Non Linéaire 7 (1990), 269-286.

[Be2] F. Bethuel, The approximation problem for Sobolev maps between two manifolds, Acta Math. 167 (1991), 153-206.

[BBC] F. Bethuel, H. Brezis, J.-M. Coron, Relaxed energies for harmonic maps, in Variational Methods (H. Berestycki, J.-M. Coron and I. Ekeland eds.), Birkhäuser, 1990, 37-52.

[BD] F. Bethuel, F. Demengel, Extensions for Sobolev maps between manifolds, Calc. Var. Partial Differential Equations 3 (1995), 475-491.

[BZ] F. Bethuel, X. Zheng, Density of Smooth Functions between Two manifolds in Sobolev Spaces, J. Funct. Anal. 80 (1988), 60-75.

[BB] J. Bourgain, H. Brezis, On the equation div $Y=f$ and application to control of phases, J. Amer. Math. Soc. 16 (2003), 393-426.

[Bo] P. Bousquet, Topological singularities in $W^{s, p}\left(\mathbb{S}^{n}, \mathbb{S}^{1}\right)$, to appear.

[BBM1] J. Bourgain, H. Brezis, P. Mironescu, Lifting in Sobolev spaces, J. Anal. Math. 80 (2000), 37-86.

[BBM2] J. Bourgain, H. Brezis, P. Mironescu, $H^{1 / 2}$ maps with values into the circle: minimal connections, lifting, and the Ginzburg-landau equation, Publ. Math. Inst. Hautes Études Sci. 99 (2004), $1-115$. 
[BBM3] J. Bourgain, H. Brezis, P. Mironescu, Lifting, Degree, and Distributional Jacobian Revisited, Comm. Pure Appl. Math. 58 (2005), 529-551.

[BBM4] J. Bourgain, H. Brezis, P. Mironescu, Density in $W^{s, p}$, in preparation.

[BBN] J. Bourgain, H. Brezis, H.-M. Nguyen, A new estimate for the topological degree, C. R. Math. Acad. Sci. Paris 340 (2005), 787-791.

[BGP] A. Boutet de Monvel-Berthier, V. Georgescu, R. Purice, Boundary Value Problem Related to the Ginzburg-Landau Model, Comm. Math. Phys. 142 (1991), 1-23.

[B1] H. Brezis, Degree theory: old and new, in Topological Nonlinear Analysis II: Degree, Singularities and Variations (M. Matzeu and A. Vignoli eds.), Birkhäuser, 1997, 87-108.

[B2] H. Brezis, New questions related to the topological degree, in Proceedings of the Conference Celebrating the 90th Birthday of I. M. Gelfand, to appear.

[BCL] H. Brezis, J.-M. Coron, E. Lieb, Harmonic maps with defects, Comm. Math. Phys. 107 (1986), 649-705.

[BL] H. Brezis, Y. Y. Li, Topology and Sobolev spaces, J. Funct. Anal. 183 (2001), 321-369.

[BLMN] H. Brezis, Y. Y. Li, P. Mironescu, L. Nirenberg, Degree and Sobolev spaces, Topol. Methods Nonlinear Anal. 13 (1999), 181-190.

[BMP] H. Brezis, P. Mironescu, A. Ponce, $W^{1,1}$-Maps with Values into $\mathbb{S}^{1}$, in Geometric Analysis and several complex variables (S. Chanillo, P. Cordaro, N. Hanges, J. Hounie and A. Meziani eds), Contemporary Mathemaircs Series, Amer. Math. Soc. 368, 2005, 69-100.

[BNI] H. Brezis, L. Nirenberg, Degree Theory and BMO. I. Compact Manifolds without Boundaries, Selecta Math. (N. S.) 1 (1995), 197-263.

[BNII] H. Brezis, L. Nirenberg, Degree Theory and BMO. II. Compact Manifolds with Boundaries, Selecta Math. (N. S.) 2 (1996), 309-367.

[C] G. Carbou, Applications harmoniques à valeurs dans un cercle, C. R. Math. Acad. Sci. Paris 314 (1992), 359-362.

[CM] R. Coifman, Y. Meyer, Une généralisation du théorème de Calderón sur l'intégrale de Cauchy, in Fourier Analysis Proc. Sem. at El Escorial, Asoc. Mat. Española, Madrid, 1980, 87-116.

[DI] J. Dávila, R. Ignat, Lifting of $B V$ functions with values in $\mathbb{S}^{1}$, C. R. Math. Acad. Sci. Paris 337 (2003), 159-164.

[E] M. Escobedo, Some remarks on the density of regular mappings in Sobolev classes of $\mathbb{S}^{M}$-valued functions, Rev. Mat. Univ. Complut. Madrid 1 (1988), 127-144.

[EM] M. Esteban, S. Müller, Sobolev maps with integer degree and applications to Skyrme's problem, Proc. Roy. Soc. London Ser. A 436 (1992), 197-201.

[FF] H. Federer, W. Fleming, Normal and integral currents, Ann. of Math. 72 (1960), 458-520.

[GMS1] M. Giaquinta, G. Modica, J. Souček, Remarks on the Degree Theory, J. Funct. Anal. 125 (1994), 172-200. 
[GMS2] M. Giaquinta, G. Modica, J. Souček, Cartesian Currents in the Calculus of Variations, II, Springer, 1998.

[HaL1] F.-B. Hang, F.-H. Lin, A remark on the Jacobians, Commun. Contemp. Math. 2 (2000), 35-46.

[HaL2] F.-B. Hang, F.-H. Lin, Topology of Sobolev mappings, II, Acta Math. 191 (2003), 55-107.

[HL] R. Hardt, F.-H. Lin, Mappings minimizing the $L^{p}$ norm of the gradient, Comm. Pure Appl. Math. 40 (1987), 556-588.

[HKL1] R. Hardt, D. Kinderlehrer, F.-H. Lin, Stable defects of minimizers of constrained variational principles, Ann. Inst. H. Poincaré Anal. Non Linéaire 5 (1988), 297-322.

[HKL2] R. Hardt, D. Kinderlehrer, F.-H. Lin, The variety of configurations of static liquid crystals, in Variational Methods (H. Berestycki, J. M. Coron, I. Ekeland, eds.), Birkhäuser, 1990, 115-131.

[JS] R. Jerrard, H. M. Soner, Functions of bounded higher variation,Indiana Univ. Math. J. 51 (2002), 645-677.

[Ka] J.-P. Kahane, Sur l'équation fonctionnelle $\int_{\mathbb{T}}(\psi(t+s)-\psi(s))^{3}=\sin t$, C. R. Math. Acad. Sci. Paris 341 (2005), 141-145.

[Ko] J. Korevaar, On a question of Brezis and Nirenberg concerning the degree of circle maps, Selecta Math. (N. S.) 5 (1999), 107-122.

[P1] A. Ponce, On the distributions of the form $\sum_{i}\left(\delta_{p_{i}}-\delta_{n_{i}}\right)$, J. Funct. Anal. 210 (2004), 391-435.

[P2] A. Ponce, personal communication.

[R] T. Rivière, Dense subsets of $H^{1 / 2}\left(\mathbb{S}^{2}, \mathbb{S}^{1}\right)$, Ann. Global Anal. Geom. 18 (2000), 517-528.

[RS] J. Rubinstein, P. Sternberg, Homotopy classification of minimizers for the Ginzburg-Landau functional in multiply connected domains, Comm. Math. Phys. 179 (1996), 257-263.

[SU] R. Schoen, K. Uhlenbeck, Boundary regularity and the Dirichlet problem for harmonic maps, J. Differential Geom. 18 (1983), 253-268.

[W] B. White, Homotopy classe in Sobolev spaces and the existence of energy minimizing maps, Acta Math. 160 (1988), 1-17. 